\title{
FERTILITY STATUS IN VISUAL PROCESSING OF MEN'S ATTRACTIVENESS
}

\author{
By \\ RAY GARZA \\ Master of Science in Psychology \\ Texas A\&M International University \\ 2011
}

Submitted to the Faculty of the

Graduate College of the

Oklahoma State University

in partial fulfillment of

the requirements for

the Degree of

MASTER OF SCIENCE

December, 2018 


\section{FERTILITY STATUS IN VISUAL PROCESSING OF MEN'S ATTRACTIVENESS}

Thesis Approved:

Dr. Jennifer Byrd-Craven

Thesis Adviser

Dr. Shelia M. Kennison

Dr. Davide Ponzi 
Name: RAY GARZA

Date of Degree: DECEMBER, 2018

Title of Study: FERTILITY STATUS IN VISUAL PROCESSING OF MEN'S ATTRACTIVENESS

\section{Major Field: PSYCHOLOGY}

Abstract: Physical characteristics are visually salient information that display an insight into a potential mate's immunocompetence, status, and reproductive potential (Djikstra \& Bunk, 2001; Dixson, Grimshaw, Ornsby, \& Dixson, 2014; Lasek \& Gaulin, 2009; Singh, 1994). In men, characteristics such as a v-shaped torso and body hair are often a desired characteristic by women because it displays men's upper body strength and hormonal levels. Recently, the use of eye tracking in attraction research has demonstrated that visual patterns are behavioral indices of interest to a potential mate. Two studies investigated visual perception of men's attractiveness across different phases of the menstrual cycle (i.e., Low vs. High Fertility). In study $1(N=83)$, men with low $(0.7)$ waist to chest ratios were rated as more attractive, and women focused most of their visual attention to the upper region of the body (i.e., head \& midriff). Study 2, $(N=44)$ replicated the findings from study 1 and found support for visual differences across the menstrual cycle using progesterone. Women viewed the head region (i.e., face) longer and took more time viewing men in general during the fertile phase of their menstrual cycle (low progesterone) compared to the non-fertile phase (high progesterone). The findings add to the existing literature on visual perception and attraction, and they contribute new findings in determining differences in visual perception across the menstrual cycle in women. 


\section{TABLE OF CONTENTS}

$\begin{array}{ll}\text { Chapter Page } & \text { Pag }\end{array}$

I. INTRODUCTION

II. REVIEW OF LITERATURE .................................................................. 3

Physical Characteristics in Mate Preferences.................................................. 3

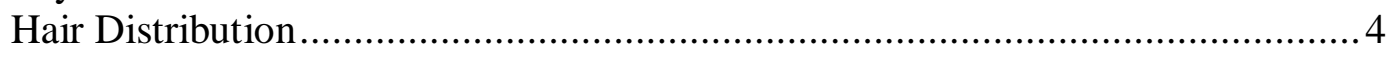

Fertility Status in Attraction ................................................................ 6

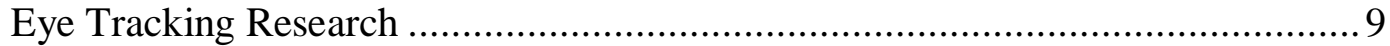

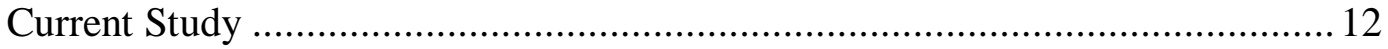

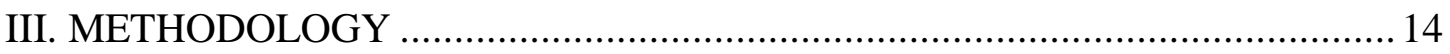

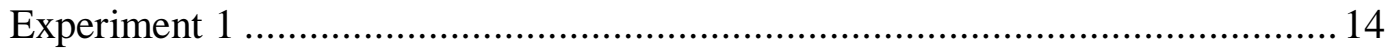

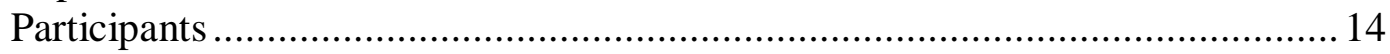

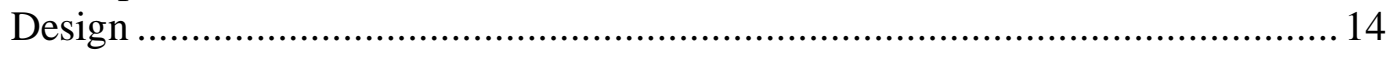

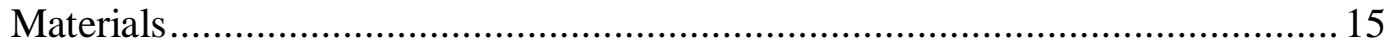

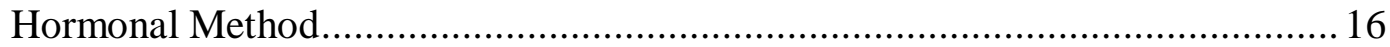

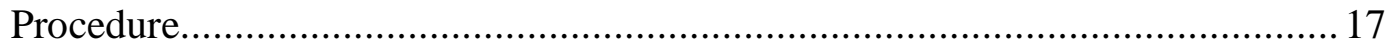




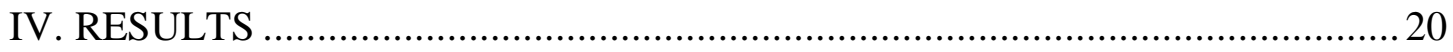

Attractiveness ........................................................................................ 20

Eye Tracking Analyses ..................................................................... 21

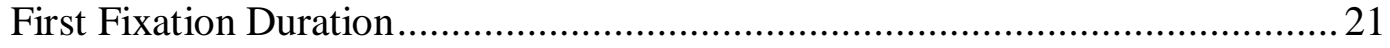

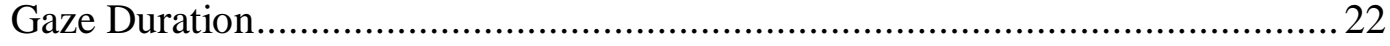

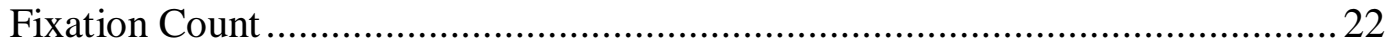

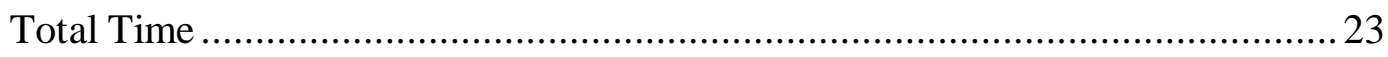

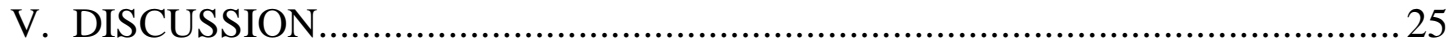

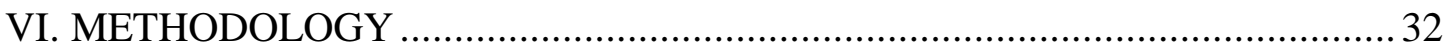

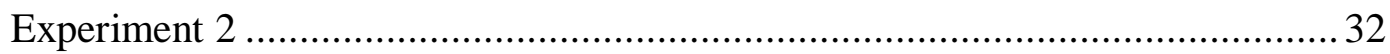

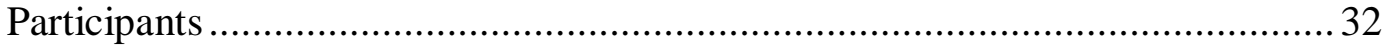

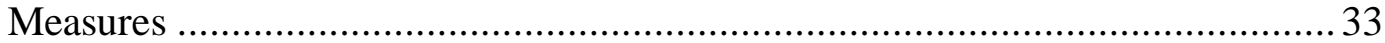

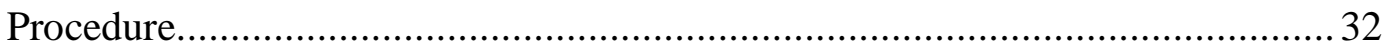




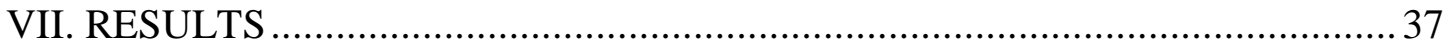

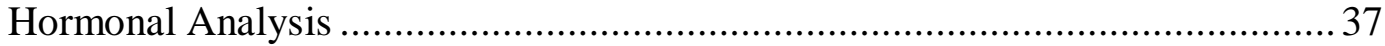

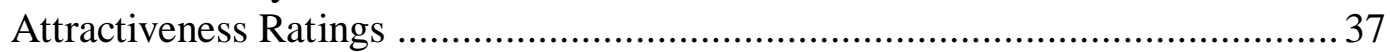

Eye Tracking Analyses ........................................................................... 38

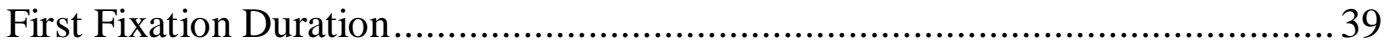

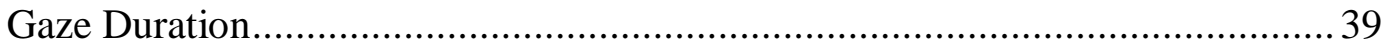

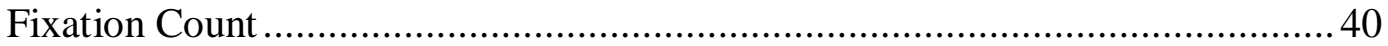

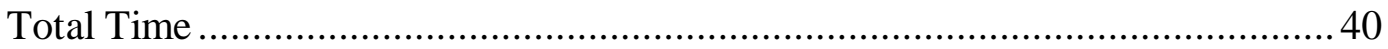

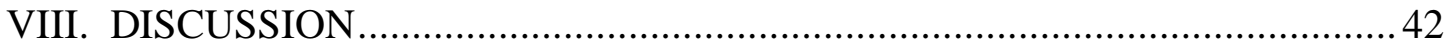

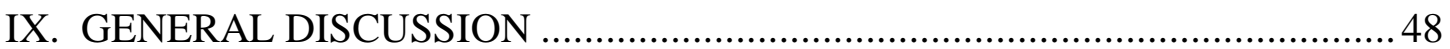

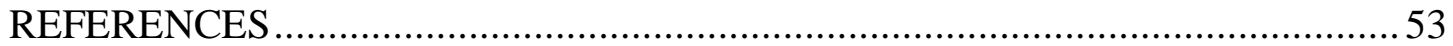

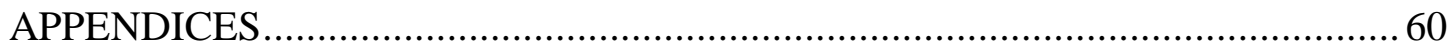




\section{LIST OF FIGURES}

Figure $\quad$ Page

Figure 1. Mean Attraction as a Function of Waist to Chest Ratio. ..........................61

Figure 2. Mean First Fixation Duration (in milliseconds) as a function of ROI: Region of

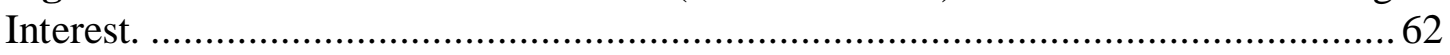

Figure 3. Mean Gaze Duration (in milliseconds) as a Function of ROI: Region of

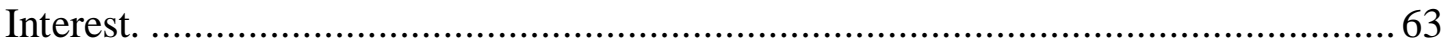

Figure 4. Mean Total Time (in milliseconds) for Waist to Chest Ratio and Hair Distribution. .64

Figure 5. Mean Attraction as a Function of Waist to Chest Ratio. 65

Figure 6. Mean First Fixation Duration (in milliseconds) as a Function of Progesterone

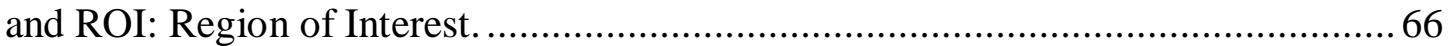
Figure 7. Mean Gaze Duration (in milliseconds) as Function of Progesterone and ROI: Region of Interest. 67

Figure 8. Mean Total Time (in milliseconds) as a Function of Progesterone levels. . 68 


\section{CHAPTER I}

\section{INTRODUCTION}

Physical characteristics are visually salient information that display an insight into a potential mate's immunocompetence, status, and reproductive potential. In men, characteristics such as a v-shaped torso and body hair are often a desired characteristic by women because it displays men's upper body strength and hormonal levels. In men, this sexual dimorphism is often referred as an android body fat distribution because fat in men is deposited in the upper-body, as opposed to women where fat is deposited in the glutofemoral region (Singh, 1993). Women perceive men that display upper body strength as more dominant, masculine, and immunocompetent (Djikstra \& Bunk, 2001; Dixson, Grimshaw, Ornsby, \& Dixson, 2014; Lasek \& Gaulin, 2009; Singh, 1994), while recent research has shown that body hair may contribute to women's perceived ratings of attraction due to its association with masculinity and hormonal differences (Dixson \& Brooks, 2013). Hair distribution is stimulated by testosterone production, specifically the conversion of testosterone to dihydrotestosterone, which begins to develop in puberty and may serve as an indirect marker of genetic fitness in mate choice (Dixson \& Vasey, 2012).

Although it is known that these physical traits are important in mate choice, 
women's menstrual cycle also plays a considerable role in choosing a mate based on fertility status (Gangestad \& Thornhill, 1998). In human populations, ovulatory cues are not obvious (i.e., cryptic) as in other species, suggesting hormonal variations may influence how sensitive women are in detecting important cues in the opposite sex (Alexander \& Noonan, 1979). Previously, attraction research depended on self-report questionnaires rating whether a man was attractive using a likert scale. Although there are benefits in using descriptive research in quantifying attractability, they fail to account for visual perception of the cues that led to making a specific rating. Research in men's attractiveness has begun using novel methods to measure women's perceived visual interest in men, such as incorporating eye tracking technology (Dixson et al., 2014; Garza, Heredia, \& Cieslicka, 2017) to specifically record the online processing of information. Nonetheless, the role that fertility status plays in visual processing of men's physical characteristics is unclear. The use of eye tracking technology to measure women's attraction to men by fertility status can provide insight into the contributing role of hormonal fluctuations in mate preferences. This insight can provide research with a specific visual framework in mate choice dependent on different phases of the menstrual cycle. 


\section{CHAPTER II}

\section{REVIEW OF LITERATURE}

\section{Physical Characteristics in Mate Preferences}

Men's physical attractiveness has often been studied by looking at obvious physical characteristics, such as body ratio differences (i.e., waist to chest/shoulder) and body hair. As in women, where waist to hip ratios can infer fertility status and health (Singh, 1994), in men, body ratio distributions have been reported to infer dominance, masculinity, increased testosterone, and immunocompetence (Braun \& Bryan, 2005, Dixson et al., 2014, Singh, 1994). Several studies have examined the waist to hip ratio (Henss, 1995), waist to shoulder ratio (Braun \& Bryan, 2006; Buunk \& Dijkstra, 2005; Hughes \& Gallup, 2002), and waist to chest ratio (Fan, Dai, Liu, \& Wu, 2005; Swami \& Tovee, 2005) as a measurement of upper body strength that is appealing in mate choice. Henss (1995) showed that men who displayed a waist to hip ratio of 0.9, which indicates a symmetrical fat distribution from the waist to hips were rated attractive compared to lower waist to hip ratios (0.7) that display more fat distribution in the hips than the waist. This suggests that increased fat distribution is more appealing to women in the upper body of men than fat distribution in the lower body (i.e., hips). Other studies using waist to shoulder or shoulder to hip ratios have shown that men who have a V-shaped torso are considered socially and physically dominant (Buunk \& Dijskstra, 2005), more likely to 
be considered for a sexual encounter (Braun \& Bryan, 2006), and have an earlier onset of sexual activities with more partners (Hughes \& Gallup, 2002). Examining a cross cultural preference, Swami et al. (2007) found that British and Greek women preferred men with lower waist to chest ratios, as they are a primary indicator of physical strength and muscular development. Research looking at somatotypes has found a distinct preference to muscular body types. Women rate mesomorphs (i.e., muscular) as more attractive than endomorphs (i.e., heavy built) (Dixson, A. F., Halliwell, East, Wingarajah, \& Anderson, 2003) because mesomorphs may provide women with greater protection from other males, resources acquisition, (Dixson et al., 2014) and possess advantages in hunting (Buss \& Schmitt, 1993). Taken together, these studies suggest that broad shoulders and narrow waists are associated with masculinity and strength, and therefore women are attracted to these characteristics in mate choice because they promote reproductive potential by advertising good genes and protection (Kasperk et al., 1997; Maisy, Vale, Cornelissen, \& Tovee, 1999).

\section{Hair Distribution}

Facial and body hair distribution are sexually dimorphic characteristics that advertise masculinity, maturity, and immunocompetence. Research on the importance of body hair distribution have been mixed, as researchers have used different means to display body hair in experimental designs. Studies using a dichotomous choice task, where women are to choose clean vs. full beard, have shown that women judge men with 
beards as older and higher in social status (Dixson \& Vasey, 2012). Women also considered men with facial hair as being more aggressive than men who were clean shaven, suggesting an important role that facial hair plays in intra-sexual competition. In mate choice, men who were clean shaven were considered more attractive than bearded men. Rantala et al. $(1999,2007)$ has suggested that clean shaven men may be considered attractive because they are less susceptible to ectoparasites, and being clean shaven may advertise muscularity in the body because hair is not concealing those features. In investigating body hair, hirsute men were judged to be older and attractive, however, that association depended on men who were considered mesomorphic (i.e., muscular) (Dixson, A. F., Halliwell, East, Wingarajah, \& Anderson, 2003). In a study investigating different categories of hirsuteness (i.e., hairless, facial, chest, \& facial/chest hair), Garza et al. (2017) did not find any significant differences in attractiveness ratings between hirsuteness categories.

Recently, studies on hair distribution have focused on degrees of hirsuteness, as opposed to a dichotomous choice. Neave and Shields (2008) demonstrated that women judged men as masculine, dominant, aggressive, and mature as the degree of facial hair increased. Interestingly, women found men who had a light stubble as attractive and preferred a light stubble for short term sexual relationships. These findings suggest that women are attracted to light stubble men because they are perceived as mature but avoid full beards because they indicate a significant increase in age (Neave \& Shields, 2008). In looking at degrees of facial and body hair, Dixson and Rantala (2016) found that men 
who had light facial hair were found to be more attractive than very light facial hair, medium, and heavy stubble beards. Body hair ratings showed different results, where men who were clean shaven were rated as most attractive. Researchers point to the depletion of body hair practice in western cultures as a contributing factor in attractiveness ratings.

\section{Fertility Status in Attraction}

A plethora of research on men's attractiveness has focused on women's fertility status because it is a woman's fertility status where reproductive advantages can be considered. During the menstrual cycle, there are optimal periods where women have the highest probability of conceiving during intercourse, known as the fertility window. Research investigating fertility status and attraction have found mixed results in whether the fertility window is an accurate predictor of being attracted to men. Most studies have used the fertility window (i.e., Days 6-14 after menstrual onset) as a means of dichotomizing women into low conceptive and high conceptive risk. Thornhill and Gangestad (1999) found that high fertile women preferred the body scent of symmetrical men compared to non-ovulating women. Penton-Voak and Perret (2000) were able to find that women preferred symmetrical faces when ovulating because symmetrical faces are associated with masculinity, which advertises hormonal levels and immunocompetence. Little, Jones, and Burriss (2007) demonstrated that women who were at the fertile period of their menstrual cycle preferred men with greater masculinity. 
Overall, these studies bring forth distinct preferences in masculine characteristics in men when the potential to pass traits to offspring is the highest (i.e., ovulation). Recently, a meta-analysis of 50 studies by Gildersleeve, Haselton, and Fales (2014) found robust support for cyclic shift preference in women. Interestingly, the effect was stronger for men who displayed cues for dominance and masculinity, however, that effect was dependent on the context, such as evaluating a mate for short term or long-term relationships. Women who were ovulating (i.e., Days 6-14 after menstrual onset) looked for cues that displayed masculinity and dominance for short term, but not long-term relationships. Preferences for masculinity and dominance could indicate a partner who is able to defend and is healthier than rival mates, particularly when the risk of conception is the highest for women. When considering relationship context, women may prefer dominant males in a short-term partner because dominance advertises social rank that is achieved through competition (Buunk, \& Dijkstra, 2005), therefore, if intercourse leads to conception, the child may inherit these qualities.

One particular study showed that fertility status may play a role in preferences for body hair. Rantala et al. (2010) presented women who were low or high conceptive risk with photographs of men who were completely shaven or hairy. The study used the traditional approach to conceptive risk (i.e., low vs. high conceptive risk) where women who were 6-14 days after the onset of their menstrual period were classified as high fertility, and women who were 0-5 and 15-28 days after the onset of their menstrual periods were classified as low fertility. Their findings demonstrated that women who 
were high fertility preferred men who were clean shaven. Rantala et al. (2010) suggests that hairiness may make men more susceptible to ectoparasites, therefore, a preference for shaven bodies indicates a lower susceptibility to parasites. In a related study, Dixson and Rantala (2016) found no effect on fertility status in preferences for facial or body hair. Replicating Dixon and Rantala (2016), Garza et al. (2017) found no preferences for facial or body hair based on fertility status. In a more recent study investigating the ovulatory shift hypothesis using accurate methods in determining fertility (i.e., hormonal assays: estradiol and progesterone) Dixson, Lee, Blake, Jasienska, and Marcinkowska (2018) found no evidence of women judging men with full beards as more attractive than clean shaven men across phases of the menstrual cycle.

An important issue with dichotomizing fertility status (i.e., low vs. high) is that it may not accurately capture women's fertility window. The most common method used when categorizing fertility status is the forward and backward counting method where researchers count the number of days from the onset of a woman's menstrual period (i.e., forward), or in reverse (i.e., backward). Both methods require knowledge and/or tracking of a woman's menstrual cycle to assess if a she is in the fertile phase of her menstrual cycle. Although convenient and inexpensive to use, accuracy of fertility is compromised, and researchers recommend using large sample sizes (i.e., $N>500$ ) when using counting methods. Blake, Dixson, O'Dean, and Denson (2016) looked at ways to increase the validity of these measurements and showed that counting methods have an accuracy of approximately $30 \%$. These findings suggest that many studies on cyclic differences have 
failed to accurately detect fertility status. Researchers suggest that to increase the validity of these measurements, more accurate means of detection should be incorporated, such as luteinizing hormone tests, estradiol, and progesterone assays (Blake et al., 2016; Gangestad et al., 2016). LH tests measure LH surges 24-48 hours preceding ovulation, therefore they are a more direct measure of cyclic changes than counting methods.

\section{Eye Tracking Research}

Eye tracking research is a novel method to use when evaluating physical attraction because it provides precise and quick information on where an individual is looking and for how long (in milliseconds). In psychology, eye tracking has been incorporated in psycholinguistic research looking at time spent on regions of interest (i.e., phrases, metaphors) when reading to indicate reading comprehension (Rayner, 1998). In attraction research, similar procedures have been used but with images to measure what areas of the human body do individuals focus most of their visual attention. Research in this field have looked primarily at women's bodies to see if there are visual paradigms that can explain where men focus most of their visual attention. Suschinsky, Elias, and Krupp (2007) asked men to rate women's bodies while manipulating waist to hip ratios (WHR) to determine if particular body types were more attractive. They found that women with lower waist to hip ratios were viewed longer and were rated as more attractive. Interestingly, they also focused on which regions of the body (ROI: regions of interest) would be more visually salient to men and showed that men made more visual 
fixations to reproductive relevant areas, such as the breasts, waists, and hips. Others (Dixson, Grimshaw, Linklater, and Dixson, 2010; Dural, Cetinkaya, \& Gulbertekin, 2008) have found similar results where men focus most of their visual attention to the upper region of women's bodies (i.e., breasts and midriff) and rate women with low waist to hip ratios as more attractive, suggesting that there are cognitive mechanisms that are used when choosing a mate.

There are only three studies that have investigated women's visual perception of men's attractiveness using eye tracking research (Anderson et al., 2010; B.J. Dixson, Grimshaw, Ormsby, \& Dixson, 2014; Garza et al., (2017). If there are cognitive mechanisms that are sensitive to specific regions, eye tracking would be able to provide a pattern of eye movements that indicate that importance. Dixson et al. (2014) manipulated men's somatotypes and presented women with back posed images of men to measure their visual time. Women spent more time viewing mesomorphs (i.e., muscular) and spent more time on the upper regions of those images suggesting that muscularity is important in visual perception. Interestingly, women who were presented with back posed men who were endomorphs (i.e., heavily built), spent more time on the lower region of the body suggesting that viewing fat distribution in the lower region may provide information as to a man's overall health. In line with Dixson et al. (2014), Garza et al. (2017) used a similar procedure, but manipulated body ratios (waist to chest ratios) and used front facing images of men. Using front faced images of men provides ecological validity to the design as women are more likely to choose a mate by viewing 
the front of the body than the rear. Their study showed that women focus most of their visual attention to the upper region of men's bodies (head, chest, \& midriff), and rate men with lower WCR as more attractive.

To date, there are only two studies that have investigated fertility status, while tracking eye movements of men's bodies (Garza et al. 2017). If women prefer specific physical traits during the time of their menstrual cycle when they are the most fertile, then their visual movements should correspond to those specific regions of the body. Anderson et al. (2010) examined how ovulation may direct attention to men's faces, and if this attention translated to better memory. Using a dichotomized measure for fertility status, women paid more attention men's faces if they were attractive, but this did not indicate better memory. The authors suggest that fertile women have fertility-enhanced attention and require a thorough examination when making an assessment. Garza et al. (2017) used the Wilcox, Dunson, Weinberg, Trussell, and Baird (2001, p. 213) likelihood of conception mean probability value to determine fertility status, as suggested by Gangestad et al. (2016), because it is a continuous measure of fertility status that is more robust in comparison to dichotomous measures. Conceptive risk was only significant for one visual analysis, total time. As conceptive risk increased, total time decreased. It is important to note that non-hormonal methods were used, and therefore cannot accurately produce actual estimates of fertility. The current study will attempt to research unanswered questions of the role that fertility status plays in visual processing using hormonal methods. 


\section{Current Study}

The current studies investigated if visual processing of men's body types is affected by fertility status. In experiment 1, Luteinizing Hormone (LH) ovulation detection sticks (e.g., Clear Blue) were used to determine fertility status, and in experiment 2, progesterone assays collected through passive drool were used in detecting fertility status. By using an eye tracker to specifically examine where women view a men's bodies, this study provides a novel framework by considering fertility status, where fertility status will be measured by hormonal methods. Based on the ovulatory shift hypothesis discussed by Gangestad and Thornhill (1998), women will be more attracted to characteristics that signal high quality. Therefore, it is hypothesized that women will demonstrate more visual interest towards men with low waist to chest ratios (WCR) when in high fertility. For experiment 1 , fertility status was determined by a LH surge, and in experiment 2 , fertility status was determined by lower progesterone levels. In experiment 2, progesterone levels will be negatively related with visual interest in men with low waist to chest ratios. Low levels of progesterone are associated with the follicular (fertile) phase of the menstrual cycle, and during this phase women should be sensitive to specific visual cues in choosing a mate (Puts et al., 2010).

Another focus of the study is whether body hair distribution will be affected by fertility status. Since research has indicated that body hair distribution in men is associated with masculinity, testosterone, maturity, and immunocompetence, eye 
movements of women were measured to determine if body hair would receive a considerable amount of visual interest when in high fertility. The second hypothesis of this study is that there will be differences in hair distribution viewing time among high fertility women. Supporting this prediction would determine that body hair distribution is a visually salient sexual ornament that men use to promote mate qualities.

Moreover, a focus of the proposed research is to see if women have cognitive mechanisms that are sensitive to specific regions of a man's body (i.e., head, chest, midriff, groin, thighs, legs/feet). As in Dixson et al. (2014, 2016), Suschinsky et al. (2007), and Garza et al. (2017), where individuals show an attentional bias towards members of the opposite sex, women will show an attentional bias towards the upper region of men's bodies when in high fertility status. These findings will suggest that there is a visual framework that women consider in mate preferences.

Lastly, aside from visual processing of men's attractiveness, the interest of the current study is to determine if men with masculine characteristics (i.e., Low WCR, Body Hair) are rated as more attractive by fertile women. This is a critical component of the ovulatory shift hypothesis because it suggests that women are not only sensitive to these physical cues visually but also place more value on men who display these characteristics. Men that display masculine physical characteristics (i.e., low WCR, Body Hair) will be rated as more attractive. 


\section{CHAPTER III}

\section{METHODOLOGY}

\section{Experiment 1}

\section{Participants}

Ninety-two undergraduate women from a Hispanic serving university from South Texas participated in the experiment. Participants were granted course credit for their participation in the study. Nine participants were excluded from the further analysis for not providing accurate information about their mensural cycle, were on hormonal based contraceptives, and were over the age of 35 . The final sample size was 83 women $(M=$ $20.75, S D=3.11)$ between the ages of 18 to 35 with menstrual cycles ranging from 25 to 35 days.

\section{Design}

The design for experiment 1 was a 2(Fertility Status: Low vs. High) X 3(WCR: 0.7, 0.8, \& 0.9) X 4(Hair: Clean, Face, Chest, \& Facial/Chest) mixed factorial design with fertility status as between-subjects, and WCR and hair as within-subjects factors. For the eye tracking analysis, a 2(Fertility Status: Low vs. High) X 3(WCR: 0.7, 0.8, \& 0.9) X 4(Hair: Clean, Face, Chest, \& Facial/Chest) X 6(Regions of Interest: Hair, Chest, 
Midriff, Groin, Thighs, and Legs/feet) with fertility status as between-subjects, and WCR, hair, and regions of interest (hereafter ROI) as within-subjects factors

\section{Materials}

A front facing photograph of a Caucasian male in his early 20's, taken from Garza et al. (2017) was used as the main stimuli. The male was manipulated using Photoshop CS6 to display different waist to chest ratios and body hair. The 3 waist to chest ratios were the following: 0.7, which displays a man with a 31 inch waist and 44 inch chest, 0.8 , which displays a man with a 35 inch waist and a 44 inch chest, and 0.9, which displays a man with a 40 inch waist and 44 inch chest. Hair distribution was manipulated to include a man with no hair, facial hair, chest hair, and both facial and chest hair. The rationale for displaying different categories of hair distribution, as opposed to hair density, is to determine which man would receive the most visual attention based on type of hair distribution, not amount. This is a novel approach as the literature has not looked at type but have focused on density. For eye tracking measurements across the body of the man, the image was divided into regions of interest. These included the head, chest, midriff, groin, thighs, and legs/feet. The head comprised of the top of the head to the man's clavicle; the chest, which was measured from the shoulder to the bottom of the man's breastbone; the midriff, which was measured from the bottom of the breastbone to the top of the man's underwear; the groin, which encompassed the pubic area; the thighs, which included upper legs to the knee; and the legs/feet, which included the bottom of the knee 
to the man's feet. In total, 12 images were used, 3(WCR: 0.7, 0.8, 0.9) X 4 Hair (Hairless, Facial, Chest, Facial/Chest).

\section{Hormonal Method}

Luteinizing Hormone. To detect fertility status in women, a clear blue digital ovulation test was used. The clear blue ovulation test detects a luteinizing hormone surge that precedes ovulation, which indicates the highest probability of conception. LH detection has been recommended by Blake et al. (2016) and Gangestad et al. (2016) as the hormonal method that yields the most accurate results in fertility status and is considered superior to counting methods and conceptive risk probability methods. The clear blue ovulation test yields a "circle" if the test does not detect a luteinizing surge, and a "happy face" if the test detects a luteinizing surge. In our sample, there were 55 women who did not detect a luteinizing surge and were classified as low fertile women, and there were 28 women who detected a luteinizing surge and were classified as high fertile women.

Menstrual Cycle Questionnaire. The menstrual cycle questionnaire asked the following: 1. "Are you on any type of contraceptive pill or medication?" 2. "Please select the first day of your last menstrual cycle?", and 3. "Please specify the typical length (in days) of your menstrual cycle.". 
Eye Tracker. The Eye-Link $1000(1000 \mathrm{~Hz})$ was used to record eye movements when processing stimuli. The visual recording was monocular (one eye), and the eye tracker was $55 \mathrm{~cm}$ from the participant. The eye tracker yielded visual recordings for first fixations (e.g., first ROI of fixation), first fixation duration (e.g., amount of time of first fixation), gaze duration (e.g., amount of time in ROI), fixation count (e.g., total number of fixations in ROI), total time (e.g., amount of time per image), and visual regressions (e.g., number of times regressed to ROI).

\section{Procedure}

Women were asked to choose a date for the study that corresponded to a date in their menstrual cycle that would detect an LH surge, which were between days 6-14 after the onset of their menstrual period. They were given consent (see Appendix I) forms that addressed the overall nature of the study, such as the samples that would be collected and the eye tracking procedure. For experiment 1, a female research assistant provided written and oral instructions on how to use the clear blue digital ovulation test. They instructed participants to remove the test strip from the test strip wrapper, insert the test strip into the tester, and urinate on the test strip only. There were two methods for LH collection using urine. One was to directly urinate on the strip for 5 seconds, and the other was to urinate in a cup, and dip the test strip for 15 seconds. Participants were given a plastic cup if they were to choose that method. Once the strip has been urinated, they were to lay the test holder with strip horizontally for 1 minute on a flat surface, and then 
return the tester with strip in a plastic bag to the laboratory. It was imperative that the tester be placed on a flat surface or the tester would yield an inaccurate or error reading. Test readings were available 5 minutes after testing had completed and yielded a dichotomous hormonal reading of low or high fertility. Once the urine sample was complete, they were directed to a computer to complete the menstrual cycle questionnaire.

The eye tracking procedure collected visual measurements form participants while they were viewed stimuli of men. Participants were instructed to sit and place their chin on a chin rest for overall stability. They were instructed that they could not move their heads once the process had begun, only their eyes. This was done to ensure that the point of fixation always captures the central field of vision throughout the entire trial. Before proceeding, participants were given overall instructions on the remote used for the experiment. They held a Microsoft Sidewinder game controller, where the left D pad button was used to proceed to the next picture, and the triggers were used to rate the attractiveness of the image (i.e., left $=$ unattractive, right $=$ attractive). The rationale for using a binary response using (i.e., No/Yes) using a game controller and not a keyboard was to prevent participants from looking down and away from the eye tracker. Participants underwent calibration and validation procedures where they would follow an "X" across the screen to ensure that their eye was recorded, and that they were following the " $\mathrm{X}$ " each time it moved. It is important to note that calibration and validation were to be completed or not the eye tracker would not proceed to the experimental trials. Once 
calibration and validation were completed, onscreen instructions informed participants of the experimental instructions. Participants were to stare at a fixation " $\mathrm{X}$ " before each picture, press the left $\mathrm{D}$ pad button to view the picture, then rate the image with the trigger options. There were a total 12 images per experiment.

Once participants were done with the eye tracker, they were asked to make numerical ratings of the images they had seen on the computer. They were presented with the same images and asked to rate the man as " $1=$ unattractive" to " $6=$ extremely attractive". This was the same continuous attractiveness measure used in Dixson et al. (2014). 


\section{CHAPTER IV}

\section{RESULTS}

Hypothesis 1 and 2 tested WCR and hair distribution as predictors in visual attention using fertility status (e.g., LH test) as a moderator. Hypothesis 3 tested if there was an attentional bias toward specific regions of interest (i.e., head, chest, midriff) among fertile women. Hypothesis 4 tested if the relationship between WCR and hair distribution as predictors in attraction was moderated by fertility status.

\section{Attractiveness}

In rating attractiveness, a 6-point likert scale was used with WCR, hair distribution, and fertility status as predictor variables. Data were analyzed with IBM SPSS V. 24. A mixed ANOVA with WCR and hair distribution as within subject variables and fertility status as between subjects variables were performed to determine if physical characteristics and fertility status predicted attraction. Mauchly's test indicated that the assumption of sphericity had been violated for hair, $X^{2}=11.31, p=.05$, therefore the Huynh-Feldt $(\varepsilon=.95)$ correction was used. The sphericity assumption was also violated for the interaction between WCR and hair, $X^{2}=33.85, p=.02$, and the HuynhFeldt correction was used $(\mathrm{e}=.96)$. The sphericity assumption was not violated for WCR, $X^{2}=1.10, p=.60$, and therefore no F-ratio correction was used for WCR. There was a 
significant main effect for WCR, $F(2,160)=126.51, p<.001, \eta_{\mathrm{p}}^{2}=.61$. Post-hoc analysis using Bonferroni's correction showed that that lowest WCR received the highest rating of attractiveness (see Figure 1). Hair was not statistically significant, $F(3,240)=$ $1.36, p=.26$. Fertility status was marginally significant in attraction ratings, $F(1,80)=$ $3.68, p=.06, \eta_{\mathrm{p}}^{2}=.04$. There was a trend for low fertile women to rate men as more attractive than high fertile women.

\section{Eye Tracking Analyses}

For the eye tracking analysis, a linear mixed effect model was conducted with fertility status, WCR, and hair distribution as fixed factors and subjects as random factors. In all eye tracking analysis, adding a random intercept variance for subjects improved the model fit, and this is represented by the $\chi 2$ statistic which compares a baseline or empty model to a random intercept model. The eye tracking data was positively skewed; therefore, a logarithmic transformation was conducted to adhere to assumptions of normality. The analyses below represent the transformed data, while the means and figures represent the untransformed data for interpretation purposes.

\section{First Fixation Duration}

First fixation duration was defined as the duration of the first fixation that was focused on a particular region of interest (ROI). Compared to a model with only a residual variance, adding a random intercept variance for subjects improved the model fit, 
$\chi^{2}=172.36, p<.001$. There was a significant main effect for ROI, $F(5,3514.23)=29.33$, $p<.001$. Follow up post-hoc analysis using Bonferroni's correction revealed that women's first fixation duration was longer for the head $(M=335.99)$ compared to the chest $(M=244.66)$, midriff $(M=288.56)$, groin $(M=222.26)$, thighs $(M=220.46)$, and the legs/feet $(M=203.84)$. There were no other significant main effects or interactions. First fixation results suggest that upon first view, the head was the area that initially attracted attention (see Figure 2).

\section{Gaze Duration}

Gaze duration was defined as the average time women spent viewing a region of interest in men. Compared to a model with only a residual variance, adding a random intercept variance for subjects improved the model fit, $\chi^{2}=1340.47, p<.001$. There was a significant main effect for ROI, $F(5,3519.02)=143.09, p<.001$. Post hoc analysis using Bonferroni's correction revealed that women spent more time viewing the midriff $(M=825.22)$ when compared to the chest $(M=578.55)$, groin $(M=281.60)$, thighs $(M=$ 394.13), and legs/feet $(M=260.59)$. Gaze duration for the midriff was not significantly longer than the head $(M=787.95)$. There were no other significant main effects or interactions (see Figure 3).

\section{Fixation Count}


Fixation count was defined as the average amount of visual fixations across regions of interest. Adding a random intercept variance for subjects significantly improved the model fit, $-\chi^{2}=5.55, p<.001$. There were significant differences in number or fixations for ROI's, $F(5,3517.01)=95.92, p<.001$. Fixation counts were higher for the upper region of the man's body. The midriff received the most visual fixations $(M=2.76)$ compared to the head $(M=2.14)$, chest $(M=2.26)$, groin $(M=1.24)$, thighs $(M=1.71)$, and legs/feet $(M=1.08)$. The interaction for WCR and ROI was significant, $F(10,3503.62)=2.03, p=.03$. The $0.9 \mathrm{WCR}$ received the most visual fixations $(M=2.86)$ compared to the $0.8 \mathrm{WCR}(M=2.77)$ and $0.9 \mathrm{WCR}(M=2.65)$. Women made more head fixations to men with the $0.9 \mathrm{WCR}(M=2.43)$ compared to the 0.7 WCR $(M=1.99)$, however fixations were not significantly different from the 0.8 $\mathrm{WCR}(M=2.00)$.

\section{Total Time}

Total time was defined as the average amount of time spent viewing each male image. A random intercept variance significantly improved the model fit compared to a residual variance, $-\chi^{2}=6.13, p<.001$. WCR, $F(2,3525.68)=7.06, p<.001$, and hair distribution, $F(3,3518.80)=66.13, p<.001$, significantly predicted the total amount of time viewing each image. Women spent more time viewing the $0.9 \mathrm{WCR}(M=2570.77)$ compared to the $0.7 \mathrm{WCR}(M=2275.60)$, and the $0.8 \mathrm{WCR}(M=2378.21)$. In reference to hair distribution, women viewed men with full body hair $(M=2737.51)$ longer than 
hairless men $(M=2214.80)$, men with facial hair $(M=2229.08)$, men with chest hair $(M$ $=2451.39)$. There was an interaction between WCR and hair distribution, $F(6,3518)=$ $7.58, p<.001$. Men with full body hair were viewed longer if they had a 0.9 WCR $(M=$ 3133.27) compared to a 0.8 WCR $(M=2677.10)$, and 0.7 WCR $(M=2402.17)$ (see figure 4). Fertility status interacted with hair distribution, $F(3,3526.83)=10.12, p<$ .001. Men with full body hair were viewed more by low fertile women $(M=2979.41)$ compared to high fertile women $(M=2495.62)$. An additional analysis was conducted to determine if binary attractive ratings (i.e., NO/YES) were influenced by physical characteristics and fertility status. Using the binary response as an independent variable, there was a significant interaction between WCR and attractive response, $F(2,3541.78)=$ $14.82, p<.001$. Women who rated the $0.9 \mathrm{WCR}$ as attractive viewed them longer than women who rated them as unattractive. Women who rated the 0.7 and 0.8 WCRs as unattractive viewed them longer compared to women who made attractive ratings. The interaction between hair and rating response was significant, $F(3,3526.31)=5.60, p=$ .001. Women viewed men longer if they had full body hair and rated them as attractive compared to women who rated full body hair men as unattractive. The interaction between fertility status and rating response was also significant, $F(1,3582.95)=9.20, p=$ .002. Low fertile women who rated men as unattractive viewed them longer, however there was no significant difference in rating time among fertile women. 


\section{CHAPTER V}

\section{DISCUSSION}

Experiment 1 investigated if attractiveness ratings and visual time was influenced by physical characteristics and fertility status. Past research has indicated men who display low waist to chest ratios are rated as more attractive because it signifies masculinity, dominance, and immunocompetence to the female perceiver (Djikstra \& Bunk, 2001; Dixson, Grimshaw, Ornsby, \& Dixson, 2014; Lasek \& Gaulin, 2009; Singh, 1994). The results of the present study showed that men with low WCRs (0.7) were rated as more attractive in comparison to the 0.8 and 0.9 WCRs. This finding partly supported hypothesis 4 , which predicted that women would find men with masculine physical characteristics (i.e., low WCR) as attractive. However, one focus of the current research was to determine if attraction was influenced by fertility status as predicted by the ovulatory shift hypothesis (Gangestad \& Thornhill, 1998). In rating men for attraction, there was a trend for low fertile women to rate men as more attractive than high fertile women, however, this finding was marginally significant. This finding contrasts with the ovulatory shift hypothesis which would suggest that women would rate men as more attractive during the fertile phase of the menstrual cycle. In reference to hair distribution, women's ratings of attraction were not influenced by where hair was distributed and fertility status. 
In investigating eye movements, visual attention was focused primarily on the upper region of men's bodies, partly supporting hypothesis 2 . The eye tracking metrics provided information as to where women looked at during the early stages of visual processing (i.e., first fixation duration \& gaze duration) and late stage processing (i.e., fixation count $\&$ total time). In the early stages of visual processing, as measured by first fixation duration, women found the head and midriff regions as salient in comparison to other ROIs. This suggested that upon first looking at men, women find the head as capturing early attention, followed by the midriff and chest region. Gaze duration lends further support to women's attentional bias to the upper region of men's bodies. Men's midriff received a considerable amount of visual attention, followed by the head region. Similar findings were also apparent in the number of fixations (i.e., fixation count). Like gaze duration, the midriff received the most visual fixations. Additionally, women made more visual fixations to the head of high WCR (0.9) men, followed by the midriff. In assessing total visual time, women viewed men with higher WCRs (0.9) and full body hair longer. This was further reinforced by viewing time and ratings made (i.e., attractive vs. not attractive) where women who rated $0.9 \mathrm{WCR}$ men as attractive viewed them longer than women who made unattractive ratings. Overall, the eye tracking data partly supported the prediction that most of the visual processing would be focused toward the upper regions of men's bodies, but it was not influenced by fertility status.

Does fertility status play a role in visual attention? There was only one eye tracking metric that demonstrated fertility effects on visual processing. Total time 
differences were apparent when presented with men with different hair distribution, where low fertile women viewed men with full body hair longer when compared to high fertile women than other hair distribution types. Therefore, fertility status does play a role in visual processing of men, however, in experiment 1 , they were in the opposite direction where low fertile women spent more time viewing.

The current findings are in line with research investigating women's mate preferences toward men who display upper body strength as measured by WCR. A low WCR may signal to women visual cues that are associated with strength, dominance, and immunocompetence, and women may consider these physical signals as determinants in high quality mates. A substantial amount of research have found that women prefer men with low WCRs in mate selection (Braun \& Bryan, 2006; Buss, 2019; Fan et al., 2005; Garza et al., 2017; Hughes \& Gallup, 2002; Swami \& Tovee, 2005) because it suggests less fat distribution in an area that is critical to one's health (i.e., midriff) (Dixson et al., 2014) and it portrays a physical appearance where upper body features (i.e., shoulders) are more apparent. The upper body features of men being important in mate selection could be a result of intrasexual competition where strength and musculature are important for physical competition, resource acquisition, and reproductive success (Barber, 1995; Genovese, 2008). Although mate preferences may be a function of fertility status, the findings in attractiveness ratings do not support the ovulatory shift hypothesis (Gangestad \& Thornhill, 1998). Given that the results were marginally significant, attraction ratings pointed in the opposite direction where low fertile women rated men as more attractive 
than high fertile women. Past research has suggested that women's mate preferences change during the ovulatory phase (Gildersleeve et al., 2014; Little et al., 2007; PentonVoak et al., 2000; Rantala et al., 2010) due to the reproductive advantages of acquiring a high-quality mate during peak fertility, however, in this study we do not lend strong support to this claim as tested using self-report ratings of attraction.

The data from eye movements supports research on visual perception suggesting that different regions of men's bodies provide informational cues to one's health. Males with more adiposity (i.e., 0.9 WCR) received a considerable amount of visual attention as highlighted by gaze duration and fixation count. Dixson et al. (2014) suggested that visual attention to the midriff region using back posed images provides information to a potential mates immunocompetence, as fat distribution in the waist is a predictor of cardiovascular disease and obesity (Janssen, Katzmarzyk, \& Ross, 2004). Although at first pass, women focused their visual attention to the head (i.e., facial region), it became apparent that at later stages (i.e., fixation count) that the midriff was viewed more. This suggests that the midriff region is an important area of men's bodies in mate preferences. The eye tracking data from hair distribution replicated findings from Garza et al. (2016) where women viewed men with full body hair and 0.9 WCRs longer. This finding is surprising given previous studies hypothesizing the importance of a low WCR (0.7), however, though not explored in this study, a high WCR (0.9) and full body hair could be a visually salient feature in determining men's overall health and maturity. It can also be a preference for a type of relationship preferences, such as a long-term mating preference, 
where physical features that advertise competition and musculature may not be a priority for women. Research by Rupp and Wallen (2007) demonstrated that normally cycling women spend more time viewing the genital region than oral contracepting women, however, in this experiment, the genitals were not a salient region of interest at the onset and throughout the stimulus presentations. All eye tracking metrics showed no support in considering the genitals visually salient. Additionally, we do not find support for Rupp and Wallen (2007) suggesting that menstrual cycle phases did not affect viewing time for regions of interest. Although not expected, the relationship was in the opposite direction where low fertile women viewed men with full body hair longer than low fertile women. The results do not find evidence raised by Anderson et al. (2010) and Maner et al. (2003), where attractive men are viewed longer by fertile women. Among attractive men, there were no significant differences in viewing time by fertile women, however, low fertile women did view unattractive men longer than attractive men.

Experiment 1 contributes to the current literature on visual perception and attraction by using an accurate measure of fertility status (i.e., ovulation detection test) in contrast to counting methods (i.e., forward and backward) that have been traditionally used in research. Using ovulation detection tests allowed for a clear grouping of fertility status dependent on the detection of a luteinizing surge, where a low and high fertile status group could be formed. Previous studies relying on counting methods may not have adequately captured fertility status in women, and if they did, the accuracy of those estimates was approximately $30 \%$ (Blake et al., 2016). The study is also the first to 
accurately study fertility status in relation to physical characteristics that are considered appealing to women (i.e., WCR and hair distribution) suing an eye tracking paradigm. Although physical characteristics have been measured using eye tracking before (i.e., Anderson et al., 2010; Dixson et al., 2014; Maner et al., 2003), they have relied on counting methods in determining fertility status, and they have utilized back posed images (Dixson et al., 2014) in contrast to the current study that used front posed images. The use of front posed images may provide an ecological stimulus in mate preferences since rating attractiveness is often accomplished by viewing a frontal view rather than a rear view of an individual. Lastly, the use of a predominantly Hispanic population widens the generalizability that can be made from women's perceptions of attractiveness. That is, cross culturally, it appears that a low WCR is a desirable attribute in mate selection.

There were a few limitations that need to be addressed for future research. First, women made general ratings of attractiveness independent of relationship preferences. Therefore, the study cannot generalize these findings to women who may have different preferences in relationships, such as short-term or long-term mate preferences. Even though women rated men with low WCRs (0.7) as more attractive, future studies can investigate if this preference is stronger for women with different relationship preferences. Given the mixed evidence on attractiveness ratings across fertility status, specifying relationship preferences could discern if preferences change across fertility status depending on relationship preferences. Another limitation to this study is that fertility status was dichotomized (i.e., Low vs. High Fertility). A continuous measure, 
such as hormonal assays (e.g., progesterone, estradiol) can provide researchers with the true relationship between fertility status and attraction. This study also used fertility status as a between subjects variable, whereas fertility status as a within subjects variable could answer the real question of within person differences in fertility status in mate preferences. Lastly, hair distribution was manipulated categorically where men had different types of body hair as opposed to length of body hair. It is very likely that the standard length used in the current study was not considered attractive, but the location was. Future research should address the length by type relationship in hair distribution.

The current study explored women's visual preferences to men's body types across the menstrual cycle. It is evident that women focus most of their visual attention to the upper region of men's bodies, but they vary as to which region when considering early and late stage processing. The findings from the eye tracking metrics lend support to the importance of the midriff region and in across WCR body types, as the midriff may hold important information about a mate's overall health. Although the study did not find high fertile women to rate men as more attractive and view them longer, the differences that were found in visual time are worth exploring when taking relationship preferences into consideration. 


\section{CHAPTER VI}

\section{METHODOLOGY}

\section{Experiment 2}

Experiment 2 examined the role of progesterone and relationship preferences while participants viewed images of men with WCR and body hair manipulations. Experiment 2 aims to answer the limitations that were raised in experiment 1, such as using a continuous measure for fertility status (i.e., progesterone) and investigating whether attraction to men is moderated by relationship preferences using the sociosexual orientation inventory. The hypotheses for experiment 2 included the hypotheses from experiment 1 with the following additions: 1). Progesterone will moderate the relationship between attraction and visual attention to men with low WCRs, where women with low levels of progesterone (i.e., high fertility) will rate men with low WCRs as more attractive and view them longer, and 2). Sociosexual orientation will moderate the relationship between attraction and visual attention, where women with unrestricted sociosexual preferences will rate men with low WCRs as more attractive and view them longer.

\section{Participants}

Forty-four undergraduate women $(M=19.56, S D=2.87)$ from Oklahoma State University participated in the experiment in exchange for course credit. Participants who 
participated were not on any form of contraceptives, did not smoke, and primarily identified themselves as heterosexual. The demographic makeup of the sample size was mostly Caucasian $(N=32)$, followed by Hispanic $(N=7)$, African-American $(N=3)$, and $A \operatorname{sian}(N=2)$.

\section{Measures}

Sociosexual Orientation Inventory. The multidimensional Sociosexual Orientation Inventory (see Appendix J), hereafter SOI (Jackson \& Kirkpatrick, 2007), was used to measure people's attitudes towards their willingness to engage in sexual intercourse $(\alpha=.77)$. A restricted sociosexual orientation is associated with a strong commitment in relationships to engage in sexual intercourse, while an unrestricted sociosexual orientation is associated with a willingness to engage in casual sex without forming any strong commitment in relationships. Participants respond to items such as, "Sex without love is ok", and "I would like to have a romantic relationship that lasts forever", in a 7-point Likert scale, where " $1=$ strongly disagree" to "7=strongly agrees". A unrestricted sociosexual orientation is associated with higher scores, while restricted sociosexual orientations are associated with lower scores.

Male Image. The male image used in experiment 1 was used for experiment 2. The male image was manipulated to display 3 WCR's $(0.7,0.8, \& 0.9)$ and 4 hair distributions (clean, facial, chest, and both: facial/chest hair). 
Progesterone. Saliva collection through passive drool was collected in $2-\mathrm{mL}$ cryovials and stored at $-80 \circ \mathrm{C}$. Saliva samples were thawed for $1 \frac{1}{2}$ hours and centrifuged at $3000 \mathrm{rpm}$ for 15 minutes before analysis. Following Salimetrics protocol on progesterone analysis, standards were serially diluted, and controls (i.e., low \& high) and samples were assayed in duplicates (i.e., $50 \mu \mathrm{L}$ ). The protocol range for the low and high controls were $1060 \mathrm{pg} / \mathrm{mL} \pm 265.03$ and low controls were $72.24 \mathrm{pg} / \mathrm{mL} \pm 28.90$.

Samples were mixed with $150 \mu \mathrm{L}$ of assay diluent diluted with a conjugate solution using a multichannel pipette. Samples were mixed for an hour at $500 \mathrm{rpm}$, then washed 4 times with deionized water in a wash buffer. Substrate stop solution $(200 \mu \mathrm{L})$ was added to each well, followed by $50 \mu \mathrm{L}$ of stop solution. The progesterone plate was read using an ELX 808 from Biotek. Intraassay coefficients of variation for progesterone were $4.14 \%$.

\section{Procedure}

Female participants who were not on hormonal based contraceptives, did not smoke, and identified primarily as heterosexual were recruited for the study. Participants signed up on an online experiment recruitment website (SONA) and signed up for 2 sessions that were at different phases of their menstrual cycle. Participants chose the first session that corresponded to the fertile phase of their menstrual cycle (i.e., follicular phase: days 6-14), and the second session that corresponded to the non-fertile phase of their menstrual cycle (i.e., days 0-5 or 14-28). Upon arrival to the laboratory, participants filled out a consent form, which was then followed by passive drool collection. Passive 
drool collection took approximately 1-5 minutes, and participants were then given a survey collecting information on demographics, menstrual cycle, and the sociosexual orientation inventory.

Once participants filled out the online surveys, they were directed to the eye tracking procedure similar to experiment 1 . Participants were instructed to maintain their visual attention on the screen using their eyes only and limit their head movement. Before presenting stimuli, a fixation validation was conducted to ensure that eye movements were being recorded. The nine-item validation procedure had to be accepted before stimulus presentation in order to adequately measure visual movement, and all participants met that requirement. Participants were instructed that they would be exposed to a male image wearing underwear only, and their task was to view the image carefully and make a binary rating (i.e., NO/YES) before presenting the next image. The rationale for using a binary rating during presentation was to limit the amount time participants directed their attention to the keyboard. A binary rating was easy to recall, and this limited the amount of time away from the screen. Each image was preceded by a fixation " $\mathrm{X}$ " to ensure visual attention was maintained at the center of the screen. As soon as all images were viewed, they were presented with the same images online where a numerical rating was made to obtain a continuous measure of attraction. Participants rated 12 male images from 1 to 6 , where " $1=$ unattractive" to " $6=$ extremely attractive". At the end of the experiment, participants were reminded to return for part 2 of the study, which was to take place during the phase of the menstrual cycle associated with low 
fertility. Session two of the experiment was identical to session one with the exception of time in the menstrual cycle. 


\section{CHAPTER VII}

\section{RESULTS}

Hypothesis 1 was tested using WCR and hair distribution as predictors and progesterone as a moderator in a linear mixed effects model. Hypothesis 2 was tested using WCR and hair distribution as predictors and sociosexual orientation as a moderator in a linear mixed effects model.

\section{Hormonal Analysis}

The hormonal analysis for progesterone was positively skewed, therefore a logarithmic transformation was conducted to adhere to assumptions of normality. Progesterone values were mean centered $(M=0, S D=1)$ and rank ordered to obtain a value for low and high progesterone across the menstrual cycle. This method for determining low and high progesterone levels was modeled after Puts et al. (2013).

\section{Attraction}

Attractiveness results were analyzed using linear mixed-effect modeling in IBM SPSS v. 24. Adding a random intercept variance significantly contributed to the model fit compared to a residual variance, $\chi^{2}=129.61, p<.01$. The fixed effects entered in to the model were WCR, hair distribution, progesterone, and SOI. There was a significant main effect for WCR, $F(2,1014.49)=588.82, p<.001$. Women rated the lowest WCR $(0.7)$ 
as more attractive $(M=3.86)$ than the $0.8 \mathrm{WCR}(M=3.01)$, and $0.9 \mathrm{WCR}(M=1.34)$ (see Figure 5). Hair distribution significant, $F(3,1014.48)=3.70, p=.01$. Pairwise comparisons showed that men with no body hair (i.e., Clean) were rated more attractive $(M=2.89)$ compared to men with full body hair $(M=2.57)$, but not significantly different than other hair distributions (e.g., facial hair and chest hair). There was a significant interaction between WCR and hair distribution, $F(6,104.48)=3.70, p=.001$ (see Figure 5). Women rated men with low WCRs (0.7) and clean-shaven bodies as the most attractive $(M=4.14)$. We investigated the role of sociosexual orientation and attraction to men's physical characteristics and found a significant interaction, $F(3$, $1014.45)=6.42, p=.003$. Women rated the lowest WCR $(0.7)$ as more attractive as they increased above the mean in sociosexual orientation (i.e., sexual unrestrictedness), $b=$ $.450, t(476.86)=2.44, p=.01$. Fertility status as measured by progesterone level was not significant, $F(1,1058.89)=3.36, p=.06$. However, there was a significant interaction between progesterone and WCR, $F(2,1014.46)=3.54, p=.02$. During high progesterone women rated men with low WCRs $(0.7)(\mathrm{M}=4.07)$ as more attractive than during low progesterone $(M=3.72)$.

\section{Eye Tracking Analyses}

For the eye tracking analysis, a linear mixed effect model was conducted with WCR, hair distribution, progesterone, and SOI as fixed factors and subjects as random factors. In all eye tracking analysis, adding a random intercept variance for subjects 
improved the model fit, and this is represented by the $\chi^{2}$ statistic which compares a baseline or empty model to a random intercept model. The eye tracking data was positively skewed; therefore, a logarithmic transformation was conducted to adhere to assumptions of normality. The analyses below represent the transformed data, while the means and figures represent the untransformed data for interpretation purposes.

\section{First Fixation Duration}

First fixation was defined as the duration of the first area fixated on, which is primarily used as an indication of saliency upon first view. Compared to an empty model with a residual variance, a random intercept variance significantly improved model fit, $\chi^{2}$ $=218.99, p<.01$. There was a significant effect for ROI, $F(5,3021.59)=37.32, p<.001$. Like experiment 1, women's first fixation duration was longer for the head region $(M$ $=473.12)$ compared to the chest $(M=358.04)$, midriff $(M=364.40)$, groin $(M=274.35)$, thighs $(M=276.93)$, and legs/feet $(M=221.10)$. There was a significant interaction between ROI and progesterone, $F(5,3014.61)=3.35, p=.005$. Women during low progesterone had longer first fixation durations for the head $(M=492.22)$ than during high progesterone $(M=452.97)$ (see Figure 6$)$. SOI interacted with ROI, $F(5,3025.19)=$ $3.10, p=.008$. As women increased above the mean on SOI (i.e., unrestricted unrestrictedness), gaze duration was longer for the head, $b=.11, t(2944.69)=2.94, p=$ .003 , and chest, $b=.17, t(2945.16)=2.89, p=.004$.

\section{Gaze Duration}


Gaze duration was defined by the average amount of time spent on ROIs. Compared to a baseline or empty model, adding a random intercept significantly improved model fit, $\chi^{2}=204.65, p<.01$. There was a significant main effect for ROI, $F(5,3035.29)=52.16, p<.001$. Women gazed at the head $(M=900.58)$, midriff $(M=$ 885.58), and chest $(M=686.24)$ longer when compared to all other regions of interest. There was a significant main effect for SOI and ROI, $F(5,3038.44)=2.34, p=.03$. As women increased above the mean on SOI (i.e., unrestricted unrestrictedness), gaze duration was longer for the head, $b=.23, t(3030.98)=2.64, p=.006$, and chest, $b=.22$, $t(3030.52)=2.54, p=.008$, regions. Progesterone negatively predicted gaze duration to the head region, $t(3029.08)=-2.31, p=.02$. Women during low progesterone gazed longer at the head regions than during high progesterone (see Figure 7).

\section{Fixation Count}

Fixation count was defined by the average amount of fixations spent on ROIs. Compared to a baseline or empty model, adding a random intercept significantly improved model fit, $\chi^{2}=179.70, p<.01$. ROI significantly predicted number of fixations, $F(5,3037.09)=48.92, p<.001$. Women made more visual fixation to the midriff $(M=$ 2.49) compared to all other ROIs. There were no other significant main effects and interactions.

\section{Total Time}


Total time measured the amount of time spent viewing the entire image independently of ROI. This allowed for an aggregate of visual time on the entire body which can be used as a measure of total attention viewing the male image. Compared to an empty baseline model, adding a random intercept model significantly improved the model fit, $\chi^{2}=360.63, p<.01$. WCR predicted total time, $F(1,957.83)=44.00, p<.001$. Women spent more time viewing the $0.7 \mathrm{WCR}(M=2362.38)$ and $0.8 \mathrm{WCR}(M=$ 2453.75) compared to the 0.9 WCR $(M=1993.14)$. Progesterone significantly predicted total visual attention, $F(1,968.26)=24.66, p<.001$. During low progesterone $(M$ $=2577.62)$ in their menstrual cycle, women spent more time viewing men than during high progesterone $(M=1961.89)$ (see figure 8). There were no other significant main effects or interactions. 


\section{CHAPTER IX}

\section{DISCUSSION}

Experiment 2 investigated attractiveness and eye movements in women throughout two different states of their menstrual cycle. Fertility status was measured with progesterone, as low levels of progesterone are associated with the follicular phase of the menstrual cycle where fertility is most optimal (Puts et al., 2013). The results from study 2 replicated study 1 where men with lower WCRs (0.7) were considered more attractive compared to other body types. In addition, women rated clean shaven men with low WCRs (0.7) as more attractive than other WCR by hair distribution combination. Although fertility status did not independently predict levels of attraction, women with high progesterone during the menstrual cycle rated men with low WCRs (0.7) as more attractive than during low progesterone. This contrasts with the ovulatory shift hypothesis (Gildersleeve et al., 2014), where fertile women are expected find men who display characteristics of dominance and masculinity as more attractive during the fertile phase of their menstrual cycle. Gildersleeve et al. (2014) suggested that the ovulatory shift may be a function of mating strategies, as fertile women may pursue short term sexual strategies with men that display desirable characteristics (i.e., low WCR). Women who were unrestricted in their sociosexual orientation found men with low WCRs as more attractive, but this was not a function of fertility status. 
The eye movement data replicated findings from study 1 . Women found the head region as the most salient area of interest upon first view, which suggests that the face region is an important area of interest when making attractiveness ratings. Additionally, as in study 1, the upper region of the man's body (i.e., head \& midriff) received a considerable amount of visual attention compared to the lower region. When making attraction ratings, women devote most of their visual attention to those regions that provide the most information as to muscularity and health. Interestingly, when taking into consideration sociosexual orientation, sexually unrestricted women (e.g., as measured through SOI) viewed the head and chest region longer. These findings suggest that shortterm mating women consider the head and chest region important when making attraction ratings. The findings from fixation count further strengthen the importance of interest in the midriff region, as the midriff region received the most visual fixations. Women may devote their visual attention to the midriff region because the midriff region is an important indicator of health, such as immunocompetence (Dixson et al., 2014).

Does fertility status predict visual attention to specific regions of interest? Eye tracking data from first fixation and gaze duration found support for fertility differences in visual processing. When women were in the fertile phase of their menstrual cycle, they viewed the head region more than when in the non-fertile phase. This region may provide important information in mate choice during the period in which women are most fertile. As has been shown with first fixation duration, in which the head region is the most salient upon first view, when women are in the fertile phase of the menstrual cycle, this 
saliency may be heightened. Another supportive finding were the total time differences in fertility status. Overall, women viewed men more when progesterone levels were low (i.e., fertile) when compared to when progesterone was high. Mate choice is a costly investment, therefore, taking more time viewing men may indicate that women are making a careful consideration before deciding on their attractiveness.

The results from physical attractiveness support previous research suggesting that low WCRs are desirable attributes in men due to the important cues they display to mates, such as muscularity, dominance, and good health (Braun \& Bryan, 2006; Buss, 2019; Fan et al., 2005; Garza et al., 2017; Hughes \& Gallup, 2002; Swami \& Tovee, 2005). Although our stimuli did not manipulate muscularity directly, manipulating WCR has been shown to give the appearance masculinity, which has been associated with lower WCRs and muscularity (Price, Kang, Dunn, \& Hopkins, 2011). Preferences for low WCR men may be a function of sociosexual orientation where attraction to men may depend on restricted or unrestricted sexuality. Provost, Troje, and Quinsey (2008) found unrestricted sexuality positively predicted preferences for masculinity, and in this study, we lend support to that claim. Unrestricted women found men with low WCRs (0.7) as more attractive compared to restricted women. Masculinity is associated with a healthy immune system and displaying these cues may signal to unrestricted women the genetic benefits the men are willing to bestow (Provost et al., 2008). 
Data from eye movements support previous research indicating that specific areas of interest are visually salient because they provide information to a mate's overall health (i.e., cardiovascular disease, immunocompetence) (Dixson et al., 2014; Garza et al., 2017; Janssen et al., 2004). Women consider the facial region (i.e., ROI: head) as most important upon first view, and they spend most of their visual attention to the upper region of men's bodies when making a rating (experiment $1 \& 2$ ). However, when looking at the whole image, women in this study viewed men with lower WCRs more than the highest WCR (0.9). This contrasts with study 1 and Garza et al. (2017), which found higher WCRs to elicit more visual time, but this could be a product of cultural differences, considering study 2 utilized a predominantly Caucasian population.

Findings from experiment 2 contribute two new findings to the existing literature on visual perception and attraction. Using an accurate method in detecting fertility (i.e., progesterone saliva collection), women's visual perception does differ based on fertility status. Women demonstrated differences in visual perception when looking at specific regions of interest and when looking at the entire image. To our knowledge, progesterone has not been used as a hormonal analysis in fertility status in detecting differences in visual perception. Therefore, it can be concluded that during the fertile phase of the menstrual cycle, women focus their visual time to specific areas (i.e., head), and spend more time viewing men before making a rating of attraction. Second, taking into consideration sociosexual orientation, visual attention was influenced by mating 
orientation. This suggests that women use specific physical cues as important information during mate choice depending on sexual restrictedness.

There are limitations to the study that need to be addressed. First, progesterone was the only hormonal analysis used in determining fertility status. To get a more accurate reading of fertility status, using progesterone and estradiol would add to the strength in approximating closeness to ovulation. Furthermore, the stimuli used in the experiment were manipulated to show variations in waist to chest ratio, and therefore all other features (i.e., shoulders, chest) were kept the same. Although it can be argued that differences were still apparent in attraction and visual attention, these regions may have still attracted attention because they were not altered to appear differently, such as adding more adiposity. Lastly, hair distribution was categorical and not quantitative. Body hair did not receive a considerable amount of visual attention, however, men with both facial and chest hair were rated as the most attractive of the set. Using degrees of hirsuteness (i.e., length) may alter the perceived ratings of attractiveness as has been shown by Dixson and Brooks (2013).

Experiment 2 extends research in visual perception and attraction by identifying the physical features that are important in mate preferences. Overall, women consider the low WCR (0.7) as the most attractive male body time and focus their visual attention on the upper region of men's bodies when making attraction ratings. The study contributes to the literature on mate preferences by demonstrating differences in visual attention 
across fertility status. 


\section{CHAPTER IX}

\section{GENERAL DISCUSSION}

Two experiments explored physical attraction to men's bodies using eye movements while tracking women's fertility status. This is the first study to use accurate methods (i.e., ovulation tests \& progesterone assays) in detecting fertility in visual perception across two different populations, Hispanic (Study 1) and Caucasian (Study 2).

In both experiments, women found men that displayed low WCRs (0.7) as more attractive, which has been a consistent finding in previous literature exploring desirable physical characteristics in men (Braun \& Bryan, 2006; Buss, 2019; Fan et al., 2005; Garza et al., 2017; Hughes \& Gallup, 2002; Swami \& Tovee, 2005). Hair distribution was not a consistent finding when rating attractiveness. In experiment 1 , hair distribution did not influence attraction ratings, however, in experiment 2 , women preferred men without no body hair (i.e., clean) as more attractive. These may reflect a cultural shift on what is considered an appealing physical trait in body hair. Cultural differences in hair distribution are not new. Previous findings have shown that British and Sri Lankan women find body hair to be physically attractive (Dixson et al., 2003), but in Chinese samples, and in a large sample using predominantly European women, clean shaven or men who displayed little body hair were preferred (Dixson et al., 2007; Dixson \& Rantala, 2016). 
Both studies replicated previous work on visual perception in men's attractiveness (Dixson et al., 2014; Garza et al., 2017). Upon first view, it is apparent that women first view the head (i.e., facial region) more before moving on to other features of men's bodies. This is in contrasts to men's perception of women's attractiveness upon first view where men focus their visual attention to the midriff region (Dixson et al., 2014; Garza et al., 2016). These findings on saliency upon first view may lend support to Singh's (1993) view of a first pass filter that is used before viewing other regions of interest. In reference to other regions of interest, women displayed an attentional bias to the upper region of men's bodies, specifically the head and midriff region. The midriff did receive a considerable amount of visual attention in both studies, therefore, the notion that women focus on that area for cues about one's overall health may be supported, as was shown in Dixson et al. (2014). When viewing the entire image, there were noticeable differences in visual attention when comparing both populations. In study 1 (Hispanic sample), women viewed men with higher WCRs (0.9) and full body hair longer, but in study 2 (Caucasian sample), women viewed men with lower WCRs (0.7 \& 0.8) longer. This may reflect cultural differences in visual attention when rating men's attractiveness, however, a follow-up study specifically addressing those cultural differences may better answer those differences.

The role of fertility status also displayed differences in visual attention, but not attraction. Fertility status did not predict attraction across the two samples, which contrasts with the ovulatory shift hypothesis (Gildersleeve et al., 2014). Nevertheless, 
there were differences in visual processing in study 2 when progesterone was used as a measure of fertility status. Women focused most of their visual attention to the head and chest region when their progesterone levels were low compared to high, and they viewed men longer before making a rating during low progesterone. These results are intriguing because they challenge the methods used in detecting cyclic shifts. With ovulation detection kits, the results are dichotomous (i.e., low vs. high fertility), but with hormonal methods such as progesterone assays, results are more quantitative, which can allow for more accurate representations of low and high fertility. The differences in fertility effects in visual perception could also be a result of research design. In study 1, a between subjects design was utilized (i.e., low fertility vs. high fertility), while in study 2, a within subjects design was used to detect variations in cyclic shifts among participants. This methodology was suggested by Gangestad et al. (2014) in which within subjects designs and hormonal analysis provide a more accurate depiction of the fertile window compared to between subjects designs.

The findings from both studies add to the existing literature on visual perception and attraction. It provides a complete visual analysis by women rating men across the menstrual cycle using two different ethnic populations. Women may have a first pass filter when viewing men before they move to other regions of interest and an attentional bias to the upper region men's bodies when making ratings. Additionally, the use of accurate methods in fertility across the menstrual cycle demonstrate that there are visual differences in regions of interest when rating men. When progesterone levels were low, 
women displayed a bias to the head and chest region and viewed men longer compared to when progesterone levels were high. In looking at sociosexual orientation, we can conclude that sociosexual orientation does influence levels of visual processing. Women who were sexually unrestricted viewed the head and chest region longer compared to sexually restricted women. For attraction ratings, unrestricted women rated low WCR (0.7) men more attractive, but in visual processing, SOI did not predict differences in visual time. To our knowledge, these two studies are the first to measure fertility status and sociosexual orientation as they relate to visual processing of men.

There are a number of suggestions for future research that should be considered. First, in detecting fertility status, using progesterone and estradiol should be used to provide a more complete depiction of the fertile window. In addition to hormonal assays, using ovulation detection kits should be considered to rule out differences in detection between the different methods. Second, in manipulating WCR, researchers may want to consider manipulating muscularity in addition to WCR to rule out if muscularity in general is to account for differences in attraction and visual time. Men with high WCR may appear to be muscular in the upper body, but not in the midriff area. These changes in stimuli can independently answer these differences. Third, hair distribution should be variable. In both studies, hair length was stable across the different types of hair distribution. As Dixson and Brooks (2013) have shown when looking at length of beards, the length does influence attraction to men. It is likely that men with full body hair were 
considered attractive because it was ideal to that sample, but there are likely to be differences in attraction when length is manipulated.

These studies bolster previous research on physical characteristics in men's attractiveness by displaying that women across two different populations find men with low WCRs as more attractive. The findings suggest that reducing adiposity in the midriff region may indicate that a mate is healthier and not susceptible to diseases compared to increased adiposity. Both studies also demonstrated that women display a first pass filter when first viewing men, as they focus most of their visual attention to the head (i.e., facial) region. The role of fertility status, primarily in experiment 2 , demonstrated that there are differences in visual attention when fertility is high compared to low fertility. This increased level of attention highlights the increased saliency that is apparent in fertile women during mate choice. 


\section{REFERENCES}

Anderson, U. S., Perea, E. F., Vaughn Becker, D., Ackerman, J. M., Shapiro, J. R., Neuberg, S. L., \& Kenrick, D. T. (2010). I only have eyes for you: Ovulation redirects attention (but not memory) to attractive men. Journal of Experimental Social Psychology, 46(5) 804-808.

Baker, R. R., \& Bellis, M. A. (1995). Human sperm competition: Copulation, masturbation and infidelity. London: Chapman and Hall.

Blake, K., Dixson, B. J. W., O’Dean, S. M., \& Denson, T. F. (2016). Standardized methodological protocols for measuring the effects of fertility on women's behavior: A data-driven approach contrasting counting and hormonal methods. Hormones and Behavior, 81, 74-83.

Braun, M. F., \& Bryan, A. (2006). Female waist to hip and male waist to shoulder ratios as determinants of romantic partner desirability. Journal of Social and Personal Relationships, 23(5), 805-819.

Buss, D. M., \& Schmitt, D. P. (1993). Sexual strategies theory: An evolutionary perspective on human mating. Psychological Review, 100, 204-232. 
Buunk, B. P., \& Dijkstra, P. (2005). A narrow waist versus broad shoulders: Sex and age differences in the jealousy-evoking characteristics of a rival's body build. Personality and Individual Differences, 39, 379-389.

Dijkstra, P., \& Buunk, B. P. (2001). Sex differences in the jealousy-evoking nature of a rival's body build. Evolution and Human Behavior, 22, 335-341.

Dixson, A., Halliwell, G., East, R., Wignarajah, P., \& Anderson, M. (2003). Masculine somatotype and hirsuteness as determinants of sexual attractiveness to women. Archives of Sexual Behavior, 32(1), 29-39.

Dixson, B. J., \& Vasey, P. L. (2012). Beards augment perceptions of men's age, social status, and aggressiveness, but not attractiveness. Behavioral Ecology, 23, 481490.

Dixson, B. J., \& Brooks, R. C. (2013). The role of facial hair in women's perceptions of men's attractiveness, health, masculinity, and parenting abilities. Evolution and Human Behavior, 34(3), 236-241.

Dixson, B. J., Tan, J. C., \& Awasthy, M. (2013). Do women's preferences for men’s facial hair change with reproductive status? Behavioral Ecology, 24(3) , 708-716.

Dixson, B. J., Grimshaw, G. M., Ormsby, D. K., \& Dixson, A. F. (2014). Eye-tracking women's preferences for men's somatotypes. Evolution and Human Behavior, 35, 73-79. 
Dixson, B. J., \& Rantala, M. J. (2016). The role of facial and body hair distribution in women's judgments of men's sexual attractiveness. Archives of Sexual Behavior, 45, 877-889.

Fan, J., Dai, W., Liu, F., \& Wu, J. (2005). Visual perception of male body attractiveness. Proceedings of the Royal Society, 272, 219-226.

Gangestad, S. W., \& Thornhill, R. (1999). Menstrual cycle variation in women's preferences for the scent of symmetrical men. Proceedings of the Royal Society B: Biological Sciences, 265, 927-933.

Gangestad, S. W., Haselton, M. G., Welling, L. M., Gildersleeve, K., Pillsworth, E. G., Burriss, R. P., Larson, C. M., \& Puts, D. A. (2016). How valid are assessments of conception probability in ovulatory cycle research? Evaluations, recommendations, and theoretical implications. Evolution and Human Behavior, 37, 85-96.

Garza, R., Heredia, R. R., \& Cieślicka, A. B. (2016). Male and female perception of physical attractiveness: An eye movement study. Evolutionary Psychology, 14(1), 1-16.

Garza, R., Heredia, R. R., \& Cieslicka, A. B. (2017). An eye tracking examination of men's attractiveness by conceptive risk women. Evolutionary Psychology, 15(1), 1-11. 
Gildersleeve, K., Haselton, M. G., \& Fales, M. R. (2014). Do women's mate preferences change across the ovulatory cycle? A meta-analytic review. Psychological Bulletin, 140, 1205-1259. doi: 10.1037/a0035438

Honekopp, J., Rudolph, U., Beier, L., Liebert, A., \& Muller, C. (2006). Physical attractiveness of face and body as indicators of physical fitness in men. Evolution and Human Behavior, 28, 106-111.

Hughes, S. M., \& Gallup Jr, G. G. (2002). Sex differences in morphological predictors of sexual behavior: Shoulder to hip and waist to hip ratios. Evolution and Human Behavior, 24, 173-178.

Little, A. C., Jones, B. C., \& Burriss, R. P. (2007). Preferences for masculinity in male bodies change across the menstrual cycle. Hormones and Behavior, 51(5), 633639. Lassek, W. D., \& Gaulin, S. J. (2009). Costs and benefits of fat-free muscle mass in men: Relationship to mating success, dietary requirements, and native immunity. Evolution and Human Behavior, 30(5), 322-328.

Lavrakas, P. L. (1975). Female preferences for male physiques. Journal of Research in Personality, 9, 324-334.

Maisey, D. S., Vale, E. L. E., Cornelissen, P. L., \& Tovee, M. J. (1999). Characteristics of male attractiveness for women. The Lancet, 353.

Maner, J. K., Kenrick, D. T., Becker, D. V., Hofer, B., Wilber., \& Neuberg, S. L. (2003). Sexually selective cognition: Beauty captures the mind of the beholder. Journal of Personality and Social Psychology, 85(6), 1107-1120. 
Mathôt, S., Schreij, D., \& Theeuwes, J. (2012). OpenSesame: An open-source, graphical experiment builder for the social sciences. Behavior Research Methods, 44(2), 314-324. doi:10.3758/s13428-011-0168-7

Neave, N., \& Shields, K. (2008). The effects of facial hair manipulation on female perceptions of attractiveness, masculinity, and dominance in male faces. Personality and Individual Differences, 45(5), 373-377.

Penke, L., \& Asendorpf, J. B. (2008). Beyond global sociosexual orientations: A more differential look at sociosexuality and its effects on courtship and romantic relationships. Journal of Personality and Social Psychology, 95, 1113-1135.

Penton-Voak, I. S., \& Perrett, D. I. (2000). Female preferences for male faces changes cyclically: Further evidence. Evolution and Human Behavior, 21, 39-48.

Puts, D., Bailey, D. H., Cardenas, R. A., Burris, P., Welling, L. L. M., Wheatley, J. R.,...Dawood, K. (2013). Women's attractiveness changes with estradiol and progesterone across the ovulatory cycle. Hormones and Behavior. 63, 13-19.

Price, M. E., Pound, N., Dunn, J., Hopkins, S., \& Kang, J. (2013). Body shape preferences: Associations with rater body shape and sociosexuality. PLOS One, $8(1), 1-9$.

Provost, M. P., Troje, N. F., \& Quinsey, V. L. (2008). Short-term mating strategies and attraction to masculinity in point-light walkers. Evolution and Human Behavior, 29, 65-69. 
Rantala, M. J., Polkki, M., \& Rantala, L. M. (2010). Preference for human male body hair changes across the menstrual cycle and menopause. Behavioral Ecology, 419-423.

Regan, P. C. (1996). Rhythms of desire: The association between menstrual cycle phases and female sexual desire. The Canadian Journal of Human Sexuality, 5(3), 145156.

Singh, D. (1993). Adaptive significance of female physical attractiveness: Role of waist to hip ratio. Journal of Personality and Social Psychology, 65, 293-307.

Singh, D. (1994). Is thin really beautiful and good? Relationship between waist to hip ratio (WHR) and female attractiveness. Personality and Individual Differences, $16,123-132$.

Singh, D. (1995). Female judgment of male attractiveness and desirability for relationships: Role of waist to hip ratio and financial status. Journal of Personality and Social Psychology, 69, 1089-1101.

Swami, V., \& Tovee, M. J. (2005). Male physical attractiveness in Britain and Malaysia: A cross-cultural study. Body Image, 2, 383-393.

Swami, V., Smith, J., Tsiokris, A., Georgiades, C., Sangareau, Y., Tovee, M. J., \& Furnham, A. (2007). Male physical attractiveness in Britain and Greece: A crosscultural study. The Journal of Social Psychology, 14(7), 15-26.

Thornhill, R., \& Gangestad, S. W. (1999). The scent of symmetry: A human sex pheromone that signals fitness? Evolution and Human Behavior, 20, 175-201. 
Wilcox, A. J., Dunson, D. B., Weinberg, C. R., Trussell, \& Baird, D. D. (2001).

Likelihood of conception with a single act of intercourse: Providing benchmark rates for assessment of post-coital contraceptive. Contraception, 63, 211-215. 


\section{APPENDICES}

\section{APPENDIX A}

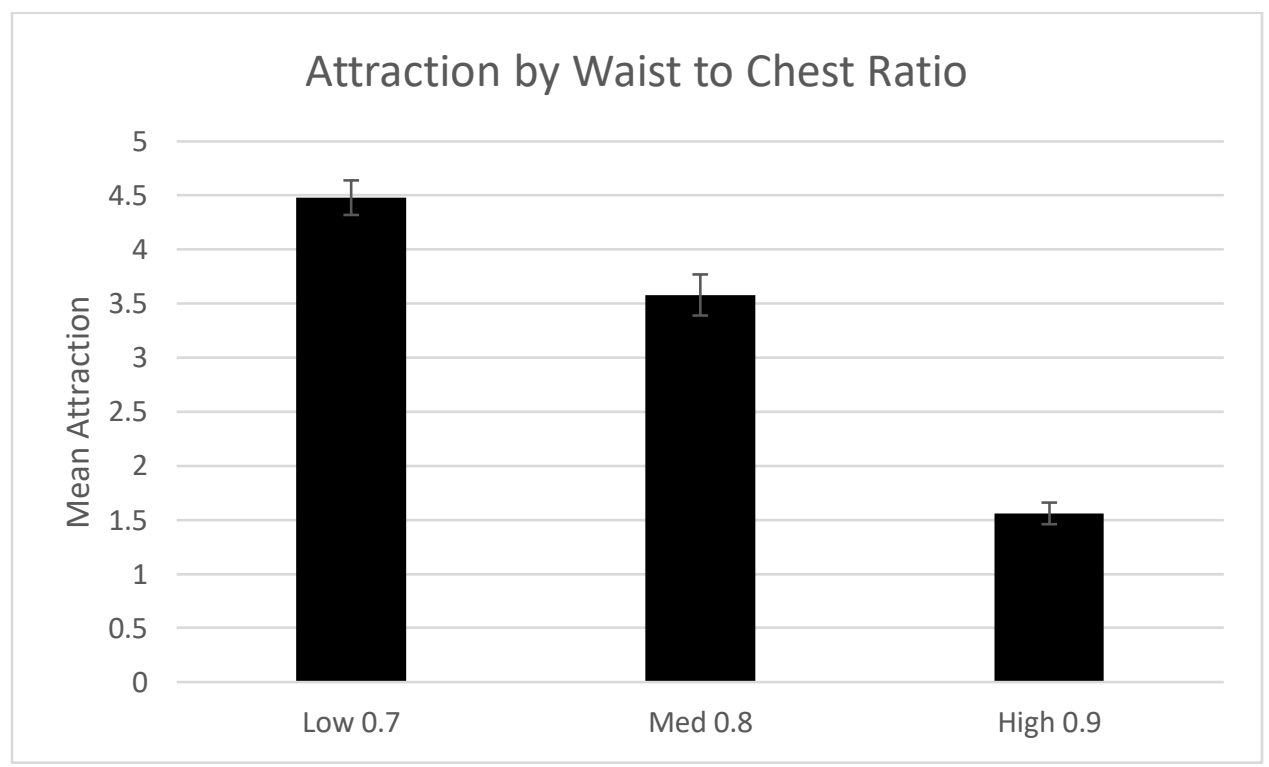

Figure 1. Mean attracting as a function of waist to chest ratio. 


\section{APPENDIX B}

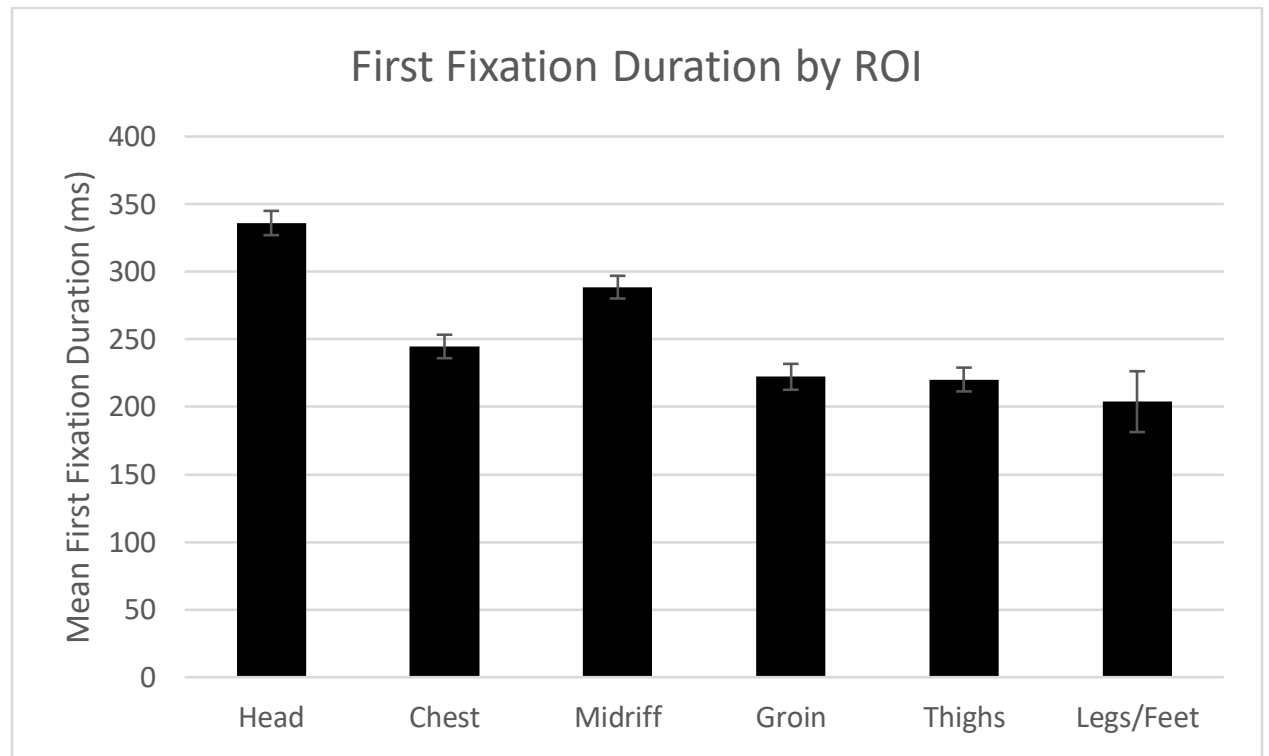

Figure 2. Mean first fixation duration (in milliseconds) as a function of ROI: Region of Interest. 


\section{APPENDIX C}

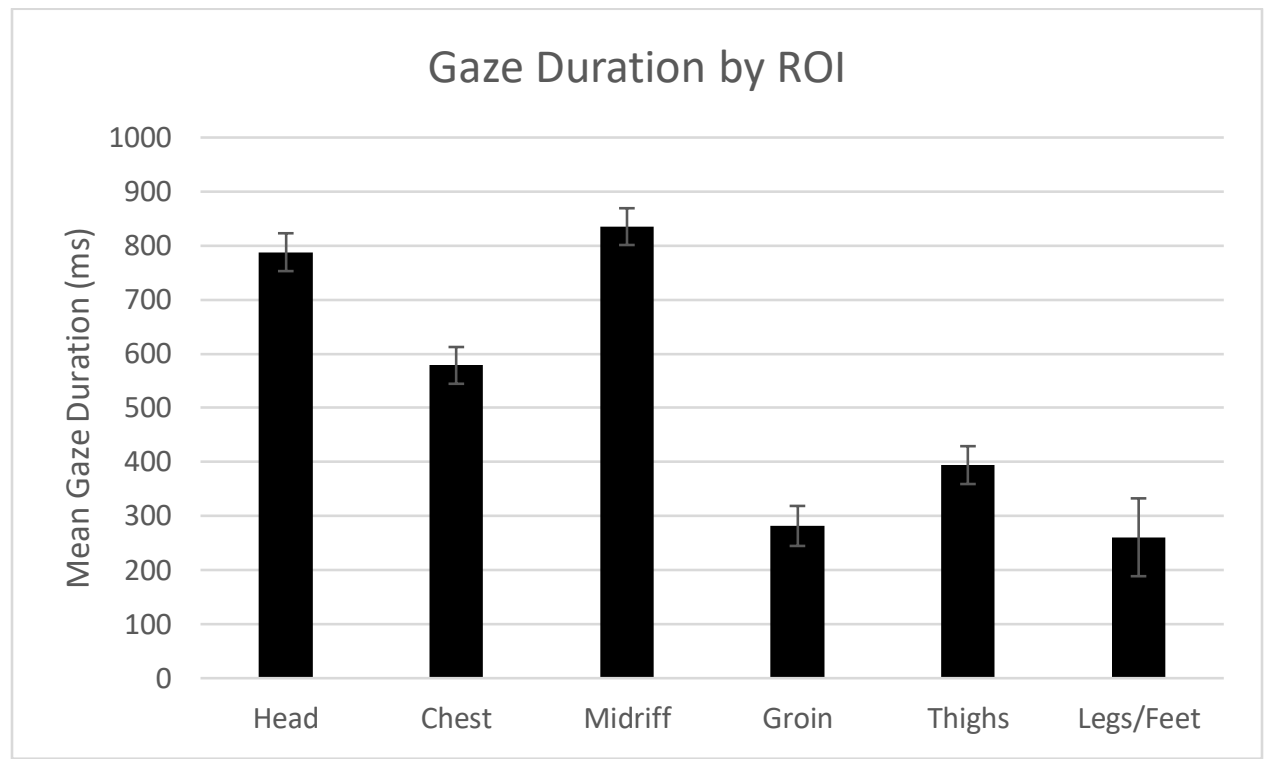

Figure 3. Mean gaze duration (in milliseconds) as a function of ROI: Region of Interest. 


\section{APPENDIX D}

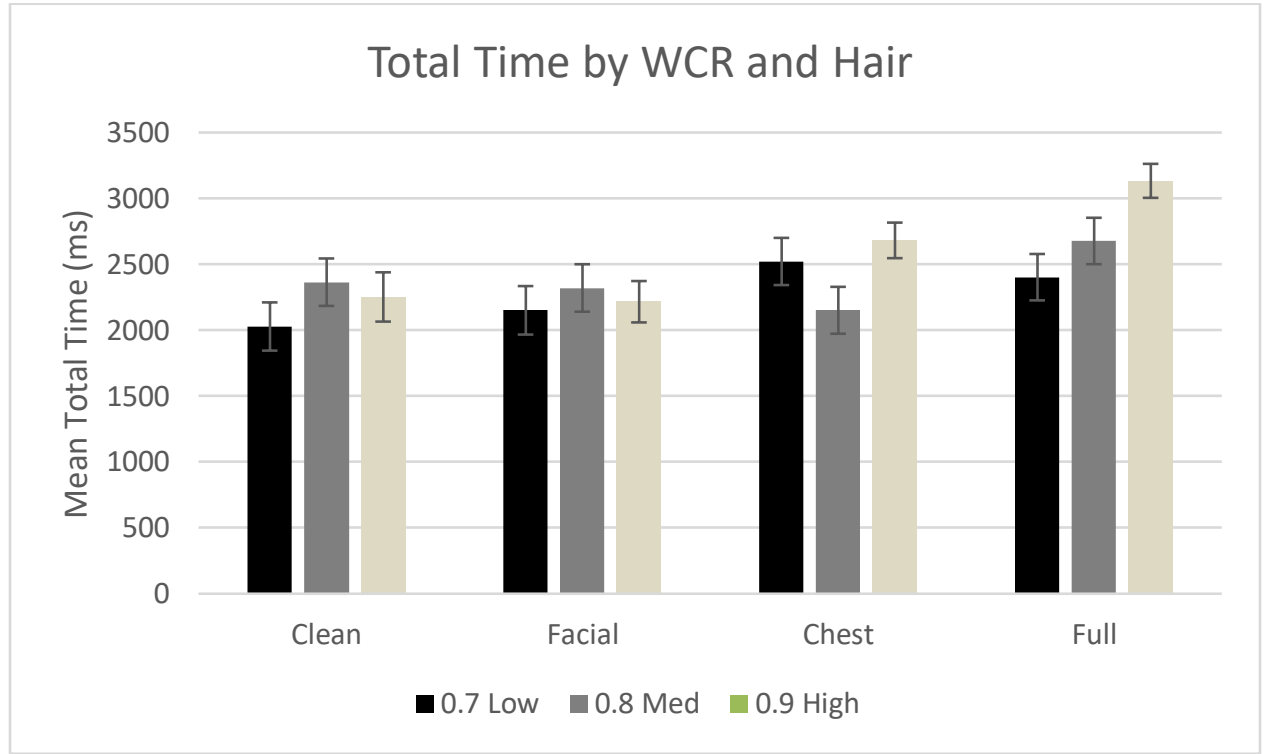

Figure 4. Mean total time (in milliseconds) for waist to chest ratio and hair distribution. 


\section{APPENDIX E}

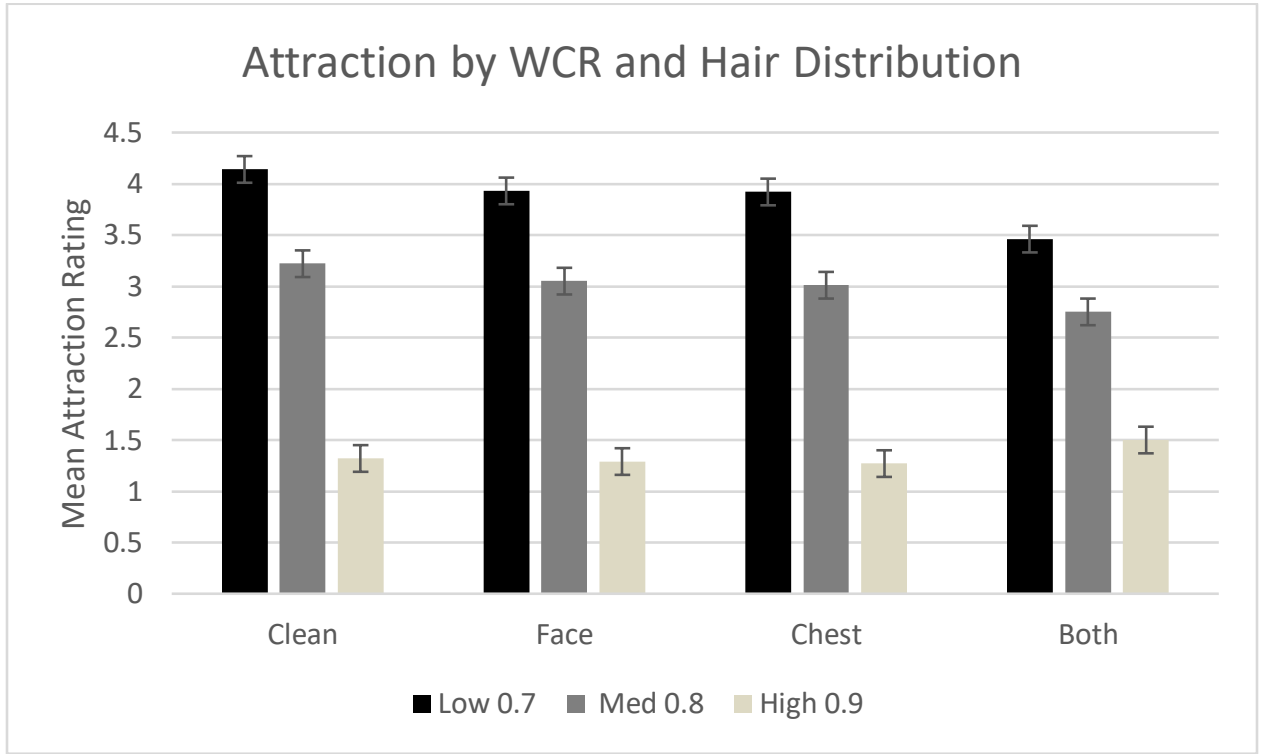

Figure 5. Mean attracting as a function of waist to chest ratio. 


\section{APPENDIX F}

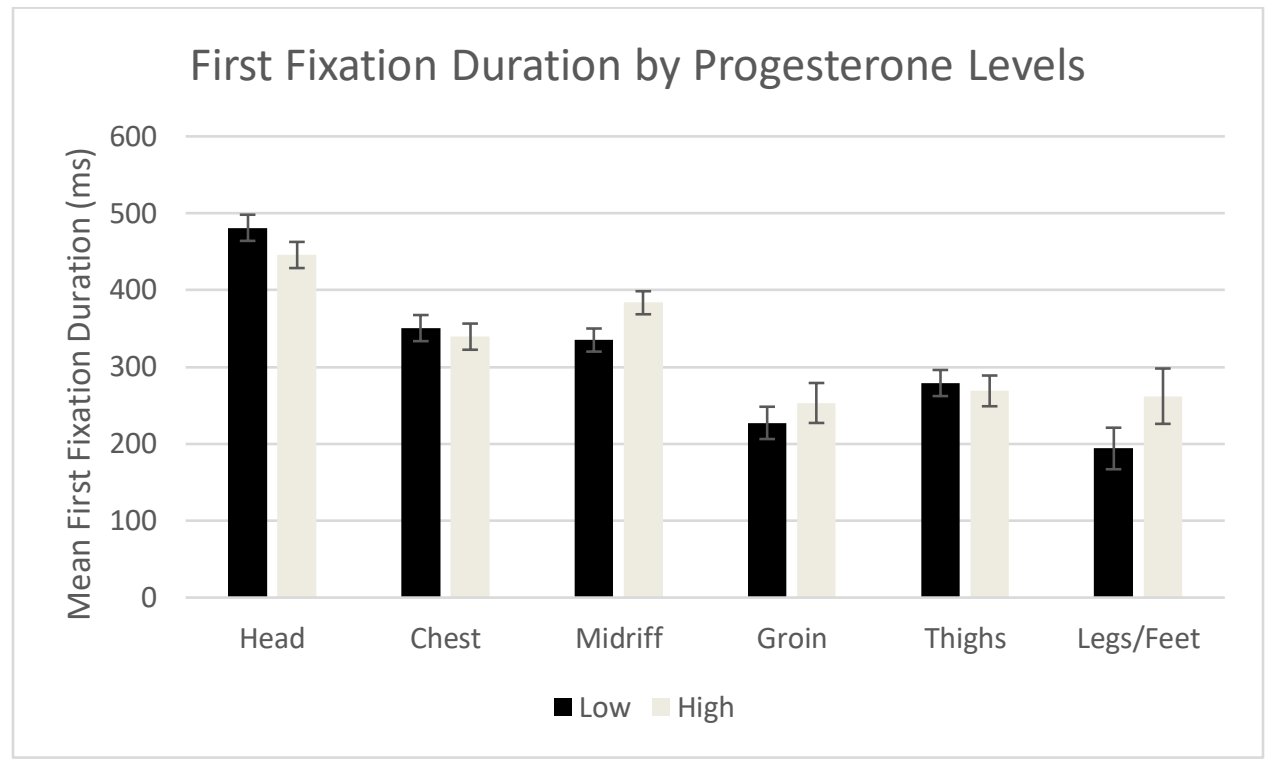

Figure 6. Mean first fixation duration (in millieseconds) as a function of Progesterone and ROI: Region of Interest. 


\section{APPENDIX G}

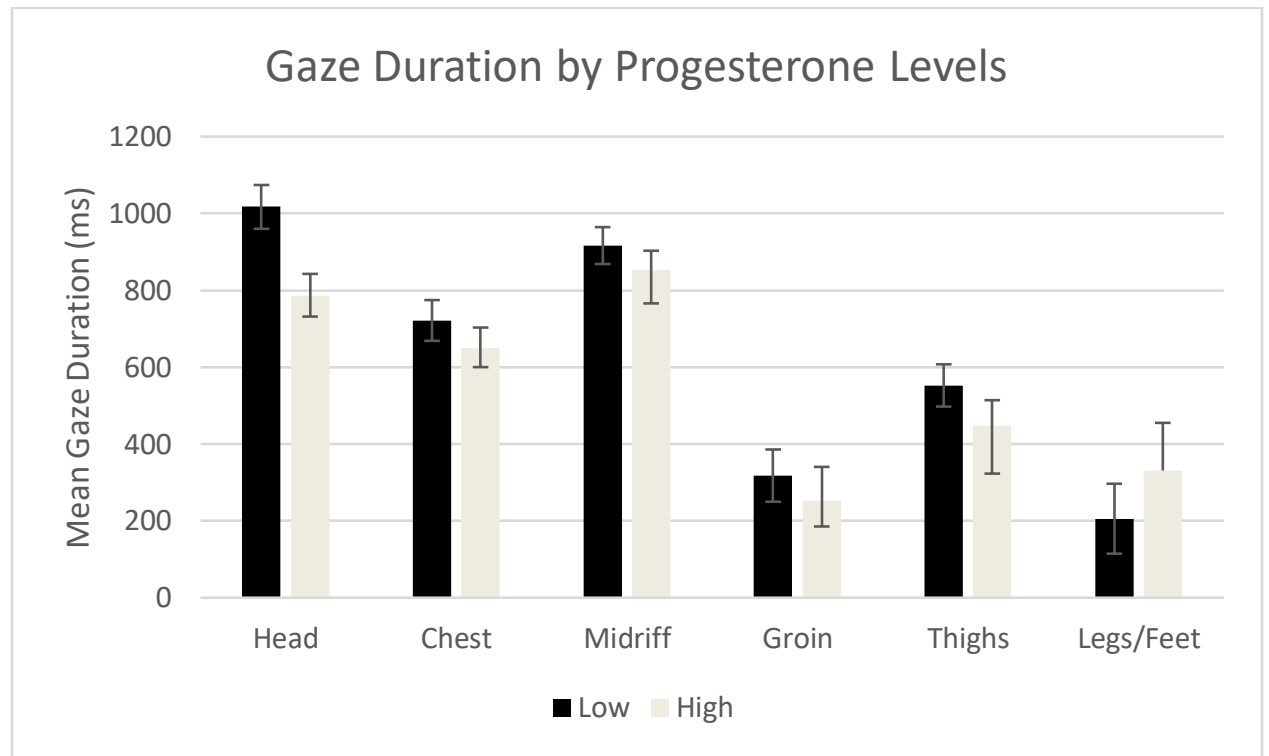

Figure 7. Mean gaze duration (in milliseconds) as function of Progesterone and ROI: Region of Interest. 


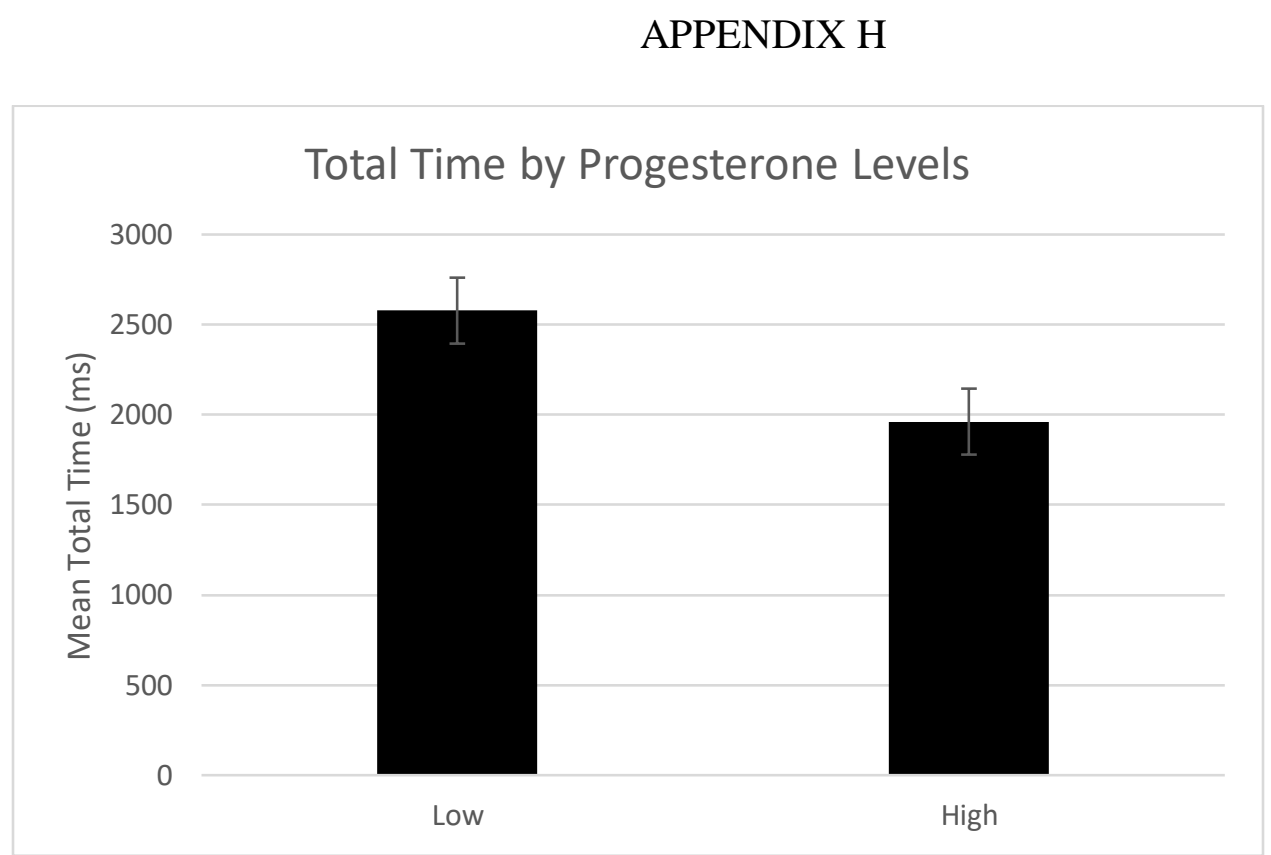

Figure 8. Mean total time (in milliseconds) as a function of Progesterone levels. 


\section{APPENDIX I}

\section{Oklahoma State University \\ Department of Psychology}

\section{CONSENT TO ACT AS A RESEARCH PARTICIPANT}

The purpose of this study is to find out more about the psychological factors contributing to our understanding of attractiveness. By signing below, you are submitting your written authorization for participation in this study.

VOLUNTARY: This study is completely voluntary. You may refuse to answer any questions or choose to withdraw from participation at any time without penalty or loss of benefits to which you are otherwise entitled.

WHAT DO YOU DO? If you agree to participate, you will be asked to participate in for one session lasting approximately one hour. The things that may happen during the study are the following:

(1)You will be asked questions about your menstrual cycle.

(2) You will be asked to submit a saliva sample. Saliva samples will be analyzed for progesterone, a hormone that naturally fluctuates throughout the menstrual cycle. Any remaining saliva after the analysis will be discarded.

(3) Your eye-movements will be measured as you scan photographs of male models wearing underwear only, taken from an online source.

(4) You will be asked to rate the attractiveness of photographs of male models wearing underwear only, taken from an online source.

(5) You will be asked to complete the MD-SOI, which is an inventory about preferences in sexual relationships.

RISKS: This project does not involve the experience of stressors greater than those encountered during everyday life or in the course of a routine medical examination. If you begin to experience discomfort or stress during this project, you may end your participation at any time.

BENEFITS: Participating in this project will not result in any direct benefits to you. However, your participation in this research study will help improve our understanding of the psychological factors contributing to our understanding of attractiveness. The experience of participating in this experiment will be educational for the field of Cognitive Science and Evolutionary Psychology.

COMPENSATION: Participants will be compensated 3 SONA credits for completing both sessions. 
CONFIDENTIALITY: The records of this study will be kept private. Your answers are completely confidential, and will not be revealed to anyone other than the researchers conducting the study. You will be given an arbitrary number for the purpose of data collection. Only this participant identification number will link you to data you provide. Therefore, your name will not appear on the survey or in the published study itself, maintaining your confidentiality. Any written results will discuss group findings and will not include information that will identify you. Research records will be stored securely and only researchers and individuals responsible for research oversight will have access to the records. There will be no future use of biospecimens as they will be discarded after analysis and will not be identifiable. It is possible that the consent process and data collection will be observed by research oversight staff responsible for safeguarding the rights and wellbeing of people who participate in research.

CONTACTS: If you have any questions about this study you may contact the researchers. They are: 1) Ray Garza, 315 N Murray Hall, Department of Psychology, Oklahoma State University, Stillwater OK, 74078, ragarza@okstate.edu; and 2) Jennifer Byrd-Craven, Ph.D., jennifer.byrd.craven@okstate.edu, (405) 744-2914, 116 North Murray Hall, Dept. of Psychology, Oklahoma State University, Stillwater, OK 74078. If you have questions about your rights as a research volunteer, you may contact the IRB office, Dr. Hugh Crethar, IRB chair, at 223 Scott Hall, Stillwater, OK, 74078, 405-7443377, orirb@okstate.edu

I have received a copy of this consent document to keep. I agree to participate in the study.

Subject's Signature

Signature of Investigator/Research Assistant

Age Date

Date 
APPENDIX J

Multi-Dimensional Sociosexual Orientation Inventory (MD-SOI, Jackson \& Kirkpatrick, 2007).

Indicate the degree to which you disagree or agree with each statement below by recording a number between " $1=$ Strongly Disagree" and " $7=$ Strongly Agree" in the space provided.

I would have to be closely attached to someone (both emotionally and psychologically) before I could feel comfortable and fully enjoy having sex with him or her.

Sex without love is ok.

I can imagine myself being comfortable and enjoying "casual" sex with different partners.

I could easily imagine myself enjoying one night of sex with someone I would never see again.

I believe in taking sexual opportunities when I find them.

I could enjoy sex with someone that I find highly desirable even if that person doesn't have long-term potential.

Sometimes I'd rather have sex with someone I didn't care about.

I would never consider having a brief sexual relationship with someone.

I can imagine myself enjoying a brief sexual encounter with someone I find very attractive. 
I would consider having sex with a stranger, if I could be assured that it was safe and $\mathrm{s} / \mathrm{he}$ was attractive to me.

I can't imagine spending the rest of my life with one sex partner.

Finding a long-term romantic partner is not important to me.

I can easily see myself engaging in a long-term romantic relationship with someone.

I would like to have at least one long-term committed relationship during my lifetime.

I would like to have a romantic relationship that lasts forever.

If I never settled down with one romantic partner, that would be okay.

I am interested in maintaining a long-term romantic relationship with someone special.

Long-term romantic relationships are not for me.

I can see myself settling down romantically with one special person.

_ I hope to have a romantic relationship that lasts the rest of my life. 
Please answer all of the following questions honestly. Please record your answers in the blank spaces and circle the appropriate number on the scales provided.

During your entire life, with how many partners of the opposite sex have you had sexual intercourse?

Of these, how many can be characterized as brief sexual relationships?

How many involved high levels of commitment on behalf of your partner?

(Commitment is defined as the desire and/or intent to maintain a relationship over the long-term.)

How many involved high levels of commitment on your part?

With how many partners of the opposite sex have you had sexual intercourse within the past year?

Of these, how many can be characterized as brief sexual relationships?

How many involved high levels of commitment (as defined above) on behalf of your partner?

How many involved high levels of commitment on your part?

With how many partners of the opposite sex do you foresee having sexual intercourse during the next five years? (Please give a specific, realistic estimate) 
Of these partners, how many do you foresee yourself having long-term, committed (as defined above) sexual relations?

Of these partners, how many do you foresee yourself having short-term, uncommitted sexual relations?

With how many partners of the opposite sex have you had sexual intercourse with on one and only one occasion?

How many times (\# of partners) have you had sexual intercourse with someone other than your relationship partner, while in a committed relationship?

How often do you fantasize about having sex with someone other than your current dating partner/spouse? (Circle One)

$1=$ Never

$2=$ Once every two or three months

$3=$ Once a month

$4=$ once every two weeks

$5=$ once a week

$6=\mathrm{a}$ few times each week

7 = nearly every day

$8=$ at least once a day 
VITA

Ray Garza

Candidate for the Degree of

Master of Science

\section{Thesis: FERTILITY STATUS IN VISUAL PROCESSING OF MEN'S ATTRACTIVNESS}

Major Field: Psychology

Biographical:

Education:

Completed the requirements for the Master of Science in Psychology at Oklahoma State University, Stillwater, Oklahoma in December, 2018.

Completed the requirements for the Master of Science in Psychology at Texas A\&M International University, December 2010.

Completed the requirements for the Bachelor of Arts in Psychology at Texas A\&M International University, August, 2009.

Experience:

Teaching

Research

Psychophysiological Data Collection and Analysis

Professional Memberships:

Association of Psychological Science

Psychonomic Society

Society for Neuroscience

Phi Kappa Phi 